\title{
NEUTRON TOTAL CROSS SECTIONS ON NUCLEI AT FERMILAB ENERGIES *
}

\author{
P.V.R. MURTHY $\ddagger$, C.A. AYRE, H.R. GUSTAFSON, \\ L.W. JONES and M.J. LONGO \\ University of Michigan, Ann Arbor, Michigan, USA, 48104
}

Received 10 March 1975

\begin{abstract}
We have measured total cross sections for neutrons on protons, deuterium, beryllium, carbon, aluminum, iron, copper, cadmium, tungsten, lead, and uranium for momenta between 30 and $300 \mathrm{GeV} / c$. The measurements were carried out in a small-angle neutral beam at Fermilab. Typical accuracy of the data is 0.5 to $1 \%$. The cross sections are consistent with an $A^{0.77 \pm 0.01}$ dependence over the entire momentum range. The cross sections are compared with theoretical predictions. Agreement is found only if inelastic screening is included. Nuclear radii obtained from our data are in good agreement with previous determinations.
\end{abstract}

\section{Introduction}

In this article we present the results of measurements of neutron total cross sections on a variety of nuclei in the momentum range 30 to $300 \mathrm{GeV} / c$. We describe the experimental technique in detail. Corrections to the data are discussed, and the results are compared to theoretical predictions. Brief accounts of this work have already been published $[1,2]$.

The cross sections were measured with a neutron beam and a technique similar to the standard good-geometry transmission technique except that an iron plate was placed just ahead of the "transmission counters" to convert the neutrons. The counters were then followed by a total absorption calorimeter which was used to measure the energy of the incident neutron. This technique for measuring neutron total cross sections was first used in an experiment by a Michigan-Princeton group at the AGS [3]. It was later used by our group in experiments at the Bevatron [4] and the AGS [5] and by other groups at CERN [6] and Serpukhov [7].

Experiments with neutron beams are the only direct method for determining np total cross sections. Measurements employing a ( $p d-p p)$ subtraction technique are

* Work supported by the National Science Foundation.

$\$$ On leave from Tata Institute for Fundamental Research, Bombay, India. 
limited to an accuracy of about $1 \mathrm{mb}$ because of uncertainties in the deuteron screening corrections *. Accurate measurements of total cross sections for heavy nuclei with charged beams are extremely difficult because of Coulomb effects. Thus, measurements with neutron beams provide the only means of answering important outstanding questions about hadron interactions in nuclei. One such question is whether screening effects neglected in the Glauber model are important at high energies ${ }^{* *}$. The comparison of the total cross section with theory is discussed in sect. 6 .

\section{Experimental arrangement}

\subsection{General description}

The measurements were carried out with scintillation uuusters and the goodgeometry transmission technique. The apparatus is shown schematically in fig. 1 . A well-collimated neutron beam containing a broad range of neutron energies is incident from the left. The monitor telescopes provide a measure of the neutron intensity incident on the target. The veto counters $A_{0}$ and $A_{1}$ in anticoincidence with the detector downstream of the target ensure that a neutral particle passed through the target into the detector.

The transmitted neutrons are detected by placing a $2.5 \mathrm{~cm}$ thick iron plate in the beam about $200 \mathrm{~m}$ downstream of the target. Charged secondaries from neutron interactions in the plate are then detected in seven circular scintillation counters $D_{1}-D_{7}$ just downstream of the plate. (The spacings of the plate and counters are exaggerated in fig. 1 for clarity.) The secondaries then enter a total absorption calorimeter which consists of iron plates totalling approximately 8 interaction lengths interspersed with scintillators. Essentially all the energy of the incident neutron is deposited in the calorimeter. The summed output from the photomultipliers viewing the scintillators is used as a measure of the neutron energy.

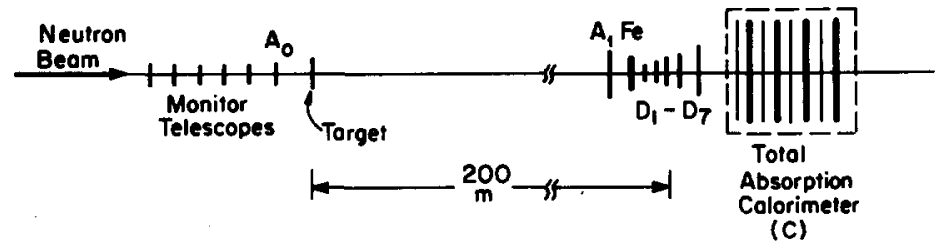

Fig. 1. Experimental arrangement (not to scale).

* For example, Carroll et al. [8] obtain a value of $0.038 \mathrm{mb}^{-1}$ for the deuteron screening parameter $\left\langle r^{-2}\right\rangle$ at $50 \mathrm{GeV} / c$, while Denisov et al. [9] use a value $0.031 \mathrm{mb}^{-1}$ in ex tracting the pn total cross section at $50 \mathrm{GeV} / c$. If Denisov et al. had used $0.038 \mathrm{mb}^{-1}$ their values for $\sigma_{\mathrm{T}}(\mathrm{pn})$ would have been $0.9 \mathrm{mb}$ larger.

** See, for example, Pumplin and Ross [27]. 
Cross sections were measured simultaneously over a broad range of neutron energies. An event was defined as a triple coincidence between two successive transmission counters and the calorimeter. The events were binned according to calorimeter pulse height to give cross sections for seven neutron energy "bins". The cross section was determined from the ratio of (events/monitor counts) with target in to that with the target out. The cross section $\sigma_{i j}$ measured by a set of two successive counters $\mathrm{D}_{i} \mathrm{D}_{i+1}$ for a particular energy bin $j$ is given by

$$
\sigma_{i j}=-\frac{1}{n x} \ln \left[\left(\frac{\sum S_{i j}}{\sum M}\right)_{\text {in }} /\left(\frac{\sum S_{i j}}{\sum M}\right)_{\text {out }}\right],
$$

where $n$ is the number of nuclei per unit volume in the target and $x$ is the target length; $\Sigma S_{i j}$ is the total number of counts recorded in a particular channel during a run, and $\Sigma M$ is the number of monitor counts during the run. To reduce systematic errors, the target was alternated between "in" and "out" about once per minute. The scaler counts were added separately for the two conditions.

As is apparent from eq. (1), the measured cross sections do not depend on the efficiency of the monitor telescopes or the neutron detector. The efficiencies cancel in the (target in/target out) ratio provided that the efficiency is the same for target in or out. Great care was taken to ensure this was the case.

If the transmission counter were vanishingly small, the cross section given in eq. (1) would be the total cross section. In practice, a correction is required for particles which scatter through such a small angle in the target that they still strike the transmission counter. This correction tends to be larger in experiments with charged beams because Coulomb scattering precludes measurements at very small angles. In our case the corrections to the cross sections measured with the smallest counter ranged from 0.2 to $7 \%$, depending on the target and the momentum range. Details of the technique used for this extrapolation are given in subsect. 4.1.

In this section we discuss in detail the beam, calorimeter, targets, beam monitors, transmission counters, and electronics. The data analysis is discussed in sect. 3.

\subsection{Beam}

The experiment was performed in the M3 neutral beam in the Meson Area at Fermi National Accelerator Laboratory. The beam line is shown schematically in fig. 2 and table 1 lists the various components in the line with their relative position and lengths. The beam is taken off at an angle of approximately $1 \mathrm{mr}$ (with respect to the incident proton beam). Most of the data were taken with $300 \mathrm{GeV} / c$ protons incident on the target; some np cross sections were also measured with $200 \mathrm{GeV} / \mathrm{c}$ protons.

This experiment was one of the first conducted in the Meson Area. Data were taken in several running periods which spanned about 18 months. During this time the performance of the accelerator improved markedly. The proton beam intensity 


\section{$M-3$ BEAM}

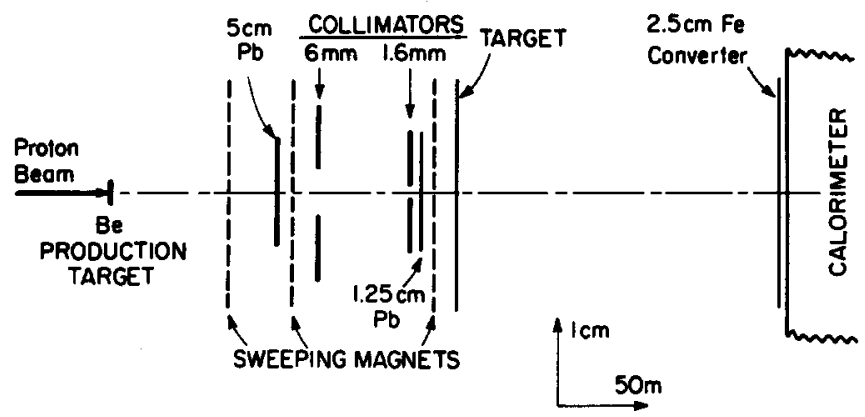

Fig. 2. Schematic diagram of the beam line. Transverse dimensions are greatly exaggerated.

Table 1

List of beam line components and their positions

\begin{tabular}{|c|c|c|}
\hline $\begin{array}{l}\text { Relative Position } \\
\text { (meters) }\end{array}$ & Component & Length \\
\hline 0 & Proton target $(3.2 \mathrm{~mm} \times 3.2 \mathrm{~mm} \mathrm{Be})$ & $30.5 \mathrm{~cm}$ \\
\hline 111.6 & Lead $\gamma$ filter & $5.1 \mathrm{~cm}$ \\
\hline 113.1 & Bending magnet & $3.0 \mathrm{~m}$ \\
\hline 122.8 & $\begin{array}{l}\text { Steel collimator, nondefining } \\
\text { (usually } 0.6 \mathrm{~cm} \text { square) }\end{array}$ & $1.2 \mathrm{~m}$ \\
\hline 196.9 & $\begin{array}{l}\text { Steel and brass defining collimator } \\
\text { (usually } 1.6 \mathrm{~mm} \mathrm{D} \text {.) }\end{array}$ & $1.5 \mathrm{~m}$ \\
\hline 197.8 & Lead $\gamma$ filter & $1.25 \mathrm{~cm}$ \\
\hline 201.2 & Two bending magnets & $6.1 \mathrm{~m}$ (total \\
\hline 205.7 & $\begin{array}{l}\text { Monitor counter telescopes } \\
(7 \text { counters) }\end{array}$ & $1.2 \mathrm{gm} / \mathrm{cm}^{2}$ \\
\hline 207.3 & Veto counter $\mathbf{A}_{0}$ & $0.2 \mathrm{gm} / \mathrm{cm}^{2}$ \\
\hline 209.4 & $\begin{array}{l}\text { Liquid hydrogen target or dummy } \\
\text { target }\end{array}$ & $121.9 \mathrm{~cm}$ \\
\hline 210.9 & Carriage for 8 solid targets & \\
\hline 399.9 & Veto counter $A_{1}$ & \\
\hline 410.6 & Transmission counters ( 7 counters) & $1.2 \mathrm{~cm}$ \\
\hline
\end{tabular}




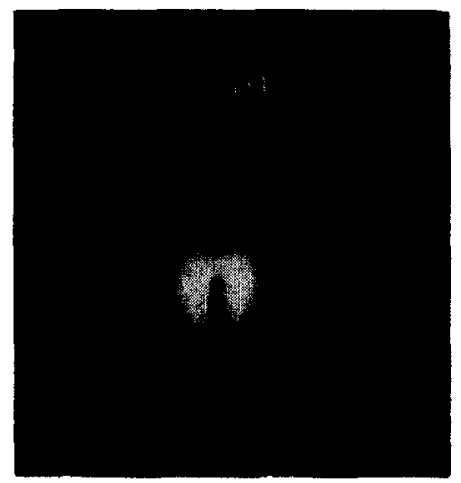

Fig. 3. Polaroid exposed in the neutron beam just ahead of the transmission counters. A $4 \mathrm{~mm}$ thick brass plate ahead of the film was used as a neu tron converter.

on the Meson Area target increased from $\sim 10^{10}$ to $\sim 10^{12}$ protons per burst. The beam spill time increased from $\sim 0.2 \mathrm{sec}$ to $\sim 1 \mathrm{sec}$, and the duty factor within this spill improved greatly. This enabled us to verify that the measured cross sections were independent of operating conditions (subsect. 4.5).

Charged particles were removed from the beam by several sweeping magnets. The majority of the high-energy photons in the beam were removed by two lead filters placed as shown in fig. 2. The beam size was defined by a steel collimator $1.5 \mathrm{~m}$ long and $197 \mathrm{~m}$ from the production target. The aperture of the collimator was $1.6 \mathrm{~mm}$ in diameter for most of the data taking. Some idea of the beam size can be obtained from fig. 3 which is a Polaroid photograph of the beam just ahead of the transmission counters taken with the aid of an intensifying screen [10]. The various targets used in the cross section measurements were placed in the beam about $3 \mathrm{~m}$ downstream of the last sweeping magnet.

\subsection{The total absorption calorimeter}

The calorimeter and its properties have been described in a previous paper [11]. Briefly, it consisted of 30 iron plates, each $30 \mathrm{gm} \mathrm{cm}^{-2}$ thick, interspersed with 30 scintillators. The total thickness of the calorimeter was about 8 interaction lengths so that essentially all of the energy of an incident hadron was deposited in the device. The scintillators sampled the energy deposited at various depths. The light output from the scintillators was optically added and brought to four 8575 photomultipliers. The summed output pulse from these was approximately proportional to the energy of the incident particle. Pulse-height distributions taken with monoenergetic proton beams of 200 and $300 \mathrm{GeV} / c$ are shown in fig. 4, along with those obtained with neutrons produced by $300 \mathrm{GeV} / c$ protons. From these and other data, it was found that the calorimeter has an energy resolution of $\pm 15 \mathrm{GeV}$ at $200 \mathrm{GeV}$ and $\pm 20 \mathrm{GeV}$ at $300 \mathrm{GeV}$ (i.e., $15 \%$ and $13 \%$ FWHM respectively). The av- 


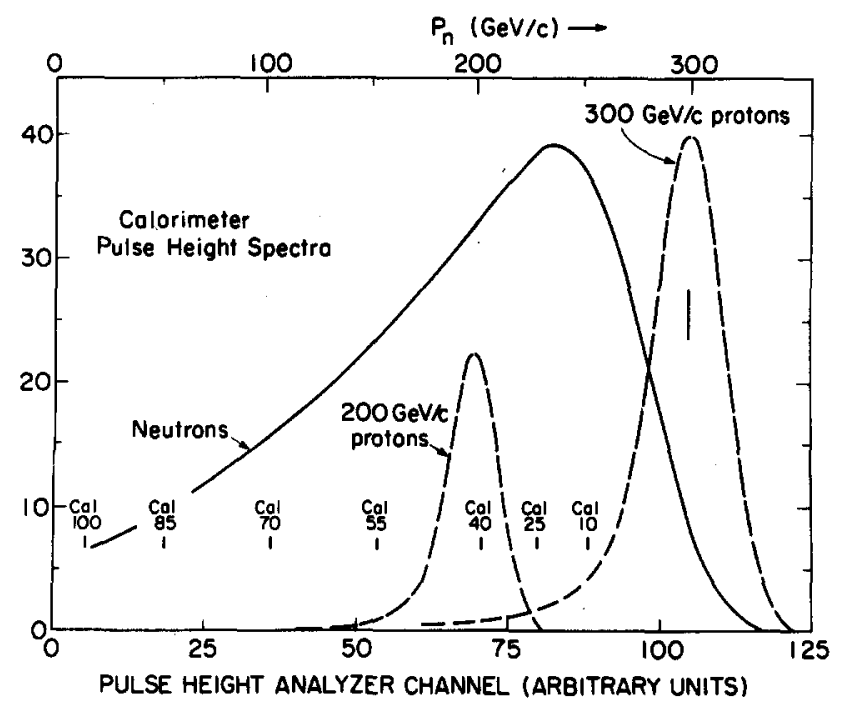

Fig. 4. Calorimeter pulse-height spectra obtained with $200 \mathrm{GeV} / c$ and $300 \mathrm{GeV} / c$ protons (dashed curves) and with neutrons (solid curve). The pulse-height cuts are also shown. The scale on top shows the average momentum corresponding to a given pulse height.

erage pulse height was found to be a linear function of incident energy to within $2 \%$. In the calibration the incident protons were required to interact in the $2.5 \mathrm{~cm}$ thick iron converter plate in front of the transmission counters so that they would closely simulate neutrons.

\subsection{The targets}

The liquid hydrogen target was a flask $1.2 \mathrm{~m}$ long and $5 \mathrm{~cm}$ in diameter operated near atmospheric pressure. Mounted next to the target in the same vacuum jacket was an evacuated dummy target. During data taking, the two targets were interchanged about once a minute. The target pressure was monitored continuously by means of a transducer connected to the target by a short tube so that the hydrogen density could be determined accurately. The pressure in the target flask was known to an accuracy of better than $0.02 \mathrm{~atm}$. which corresponds to an uncertainty of $0.1 \%$ in hydrogen density. The hydrogen could be viewed through the mylar end windows. No significant bubbling was observed in that part of the vessel illuminated by the beam. The target length was measured to an accuracy of $\pm 0.5 \mathrm{~mm}$ at room temperature and at liquid nitrogen temperature. A small correction $(-2.5 \mathrm{~mm})$ was then made to determine the length at liquid hydrogen temperature. The flask was constructed of an alloy of aluminum whose coefficient of expansion is well known, so this correction could be made quite accurately. During the deuterium runs, the hy- 
drogen was replaced with liquid deuterium. Hydrogen and deuterium densities and vapor pressures were taken from a recent NBS compilation [12].

The hydrogen had a purity of $99.99 \%$. The deuterium had a contamination of $0.3 \%$ by weight of HD. Sufficient time elapsed between filling the target with hydrogen and data taking to ensure that the (ortho $\rightarrow$ para) conversion was essentially complete, so the hydrogen was almost pure parahydrogen [12]. The conversion of parato orthodeuterium requires $\sim 10^{4}$ hrs, so the density for normal deuterium was used *. As a check, cross sections were measured with no hydrogen in the normally full target. As expected, the cross sections were consistent with zero. The uncertainty in the total cross section due to the uncertainty in the target length and density is estimated to be $<0.2 \%$ for hydrogen and $<0.35 \%$ for deuterium.

The solid targets were mounted on a platform slightly downstream of the hydrogen target. The platform was designed to hold eight targets; one or more could be placed in or out of the beam by a command from the online computer controlling the experiment. The solid targets were carefully machined blocks with transverse dimensions $\sim 8 \mathrm{~cm}$. The thicknesses were chosen to give an attenuation between 20 and $30 \%$. The density per unit area of the solid targets was known to better than $0.1 \%$. All solid targets were naturally occurring isotopic mixtures except the uranium which was $\mathrm{U}^{238}$.

\subsection{The beam monitors}

Two independent beam monitor counter telescopes were mounted upstream of the hydrogen target. Each consisted of a veto counter followed by a polyethylene converter and 3 scintillators in triple coincidence. These were used as a measure of the intensity of the neutron beam. As discussed in subsect. 2.1, the efficiency of the monitors need not be known to determine cross sections. Two monitor telescopes were used for redundancy. Different types of phototubes were used in the two telescopes to provide an additional check that the cross sections did not depend on the characteristics of the monitor. No significant differences were found between cross sections determined with the two monitors.

Placed immediately downstream of the monitors was a small scintillation counter, $\mathbf{A}_{0}$. It was used as a veto counter to ensure that no charged particles entering the target could be recorded as neutrons.

\subsection{The transmission counters}

Seven circular transmission counters $\left(D_{1}-D_{7}\right)$ with radii between 1 and $5 \mathrm{~cm}$ were placed just in front of the calorimeter. These counters were constructed of a $1.6 \mathrm{~mm}$ scintillator disc mounted in a square lucite piece. This mode of construction

* For hydrogen the para and normal forms differ in density by about $0.26 \%$; for deuterium this difference is only $0.11 \%$ (ref. [12]). 
ensured uniform light collection from the whole disc. Light from each scintillator was brought to a 56AVP photomultiplier by a lucite light pipe, $1.6 \mathrm{~mm}$ thick. The light pipes came off in different directions to minimize coincidences due to Cerenkov light from the light pipes. Each of the scintillators was surrounded by lucite of the same thickness as the counter so that the amount of converter seen by a neutron was not a function of radius. The smallest counter was in contact with the $2.5 \mathrm{~cm}$ thick iron converter and the largest was about $1.3 \mathrm{~cm}$ away. Charged particles formed by neutron interactions in the iron tend to go nearly along the direction of the incident neutron so that the transmission counters saw a charged particle distribution which closely approximated the spatial distribution of the transmitted neutrons at the iron converter. This is discussed further in subsect. 4.1. The counters were centered on the beam to within $\pm 1 \mathrm{~mm}$ by means of Polaroid exposures. A coincidence between two successive counters was always required to reduce accidental rates. The smaller counter of course determined the effective size of the pairs. Thus, there were effectively only six transmission counters. The high voltages of the transmission counters were set with a beta source so that a pulse approximately 1.4 times that from a minimum ionizing particle was required from the counter. Thus on the average two or more charged particles had to pass through the scintillator for a neutron to be counted.

A second veto counter, $A_{1}$, was positioned about $11 \mathrm{~m}$ upstream of the transmission counters to ensure that the particle that entered the iron converter was neutral.

\subsection{Electronics}

Most of the fast electronics used in the experiment were conventional modules manufactured commercially. A total of 64 scalers recorded the data. The scaler readings were written on magnetic tape after each beam pulse as explained below. Forty two of these scaled coincidences were of the type $\bar{A}_{0} \bar{A}_{1} D_{i} D_{i+1} C_{j}$ where $A_{0}$ and $A_{1}$ are the veto counters and $C_{j}$ represents a pulse from one of seven discriminators which were set to trigger only if the pulse height from the calorimeter exceeded some minimum value $\delta_{j}$. The $\delta_{j}$ corresponded to energies deposited in the calorimeter of approximately $14,52,104,154,206,231$ and $252 \mathrm{GeV}$, as determined from the calibration with protons (see fig. 4). The other twenty-two scaler channels recorded beam monitors, accidental coincidences of various kinds, single rates, and proton beam intensity.

The events were divided into seven momentum bins by subtracting counts in successive momentum ranges. The nominal momentum ranges and central momenta for these bins are given in table 2 . These were determined from pulse-height spectra of events passed by each of the discriminators. The uncertainties in the central momenta are approximately $\pm 5 \mathrm{GeV}$.

The scaler values were recorded after each beam pulse on magnetic tape by a PDP$11 / 20$ computer. The targets were interchanged by the computer whenever the count from one of the monitors reached a predetermined value which was usually 
Table 2

Nominal momentum ranges and central momenta for the seven neutron momentum bins

\begin{tabular}{|ccc|}
\hline & Momentum Range $(\mathrm{GeV} / \mathrm{c})$ \\
\hline Min. & Max. & $\frac{\text { Mean }}{34}$ \\
\hline 14 & 52 & 80 \\
52 & 104 & 131 \\
104 & 154 & 180 \\
154 & 206 & 215 \\
206 & 231 & 240 \\
231 & 252 & 273 \\
252 & 300 & \\
\hline
\end{tabular}

set so that the change occurred after about 6 beam pulses, or about once per minute. Data with a given target were taken in runs lasting from 30 to 90 minutes. Between 6 and 80 runs were taken with each target. Typically about $2 \times 10^{4}$ neutrons were incident on the target each accelerator pulse. Of these, approximately $20 \%$ interacted in the target. About $15 \%$ of the neutrons interacted in the iron converter ahead of the transmission counters. In a typical run $\sim 2 \times 10^{5}$ events were recorded in each momentum bin.

\section{Data analysis}

The data were analyzed online by the PDP-11 computer. The online analysis provided a check on equipment operation and on overall consistency. The final analysis was done offline on a PDP-10 computer. The data were first checked for consistency at the beam pulse-to-beam pulse level. Occasional bad data were edited as described below. The edited runs were then put on a summary tape. Another program was then used to check for run-to-run consistency and to average data from various runs. Cross sections were obtained from eq. (1). For a given momentum range, cross sections for each of the six $D$ counter combinations $\left(D_{1} D_{2}, D_{2} D_{3}, D_{3} D_{4}, D_{4} D_{5}, D_{5} D_{6}\right.$, and $\mathrm{D}_{6} \mathrm{D}_{7}$ ) were obtained from the data in a given run. This set of so-called partial cross sections is used to obtain the total cross section as described in subsect. 4.1. 


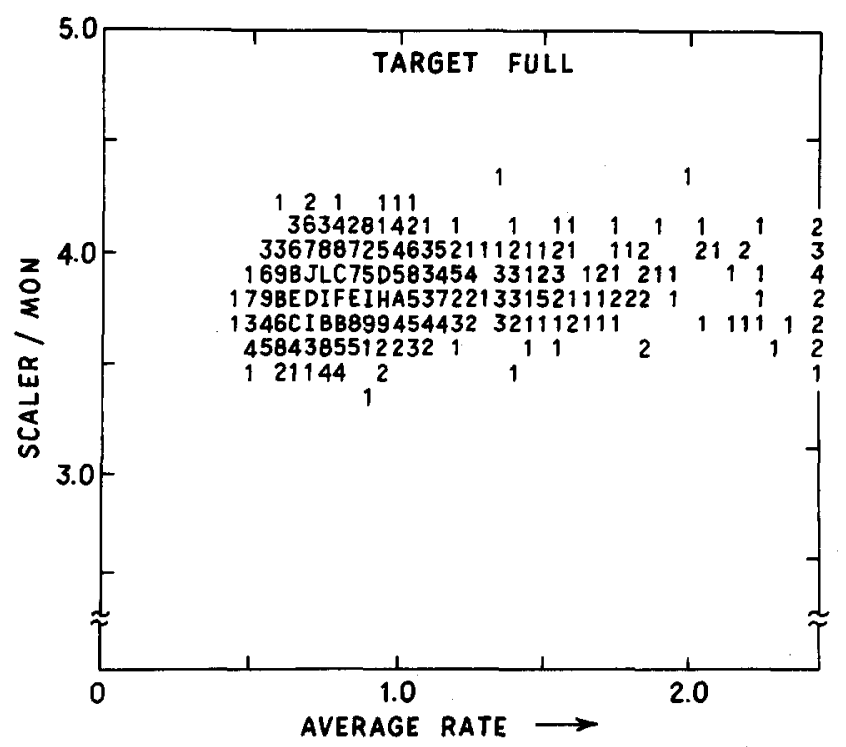

Fig. 5. Typical scatter plot showing the scaler/monitor ratio versus the average instantaneous counting rate for each beam pulse during a run. The vertical spread is consistent with expected statistical fluctuations in the ratio.

\subsection{Data editing}

Occasionally the ratio of calorimeter counts to monitor counts was observed to change abruptly during a run. This was usually due to a known cause such as a sweeping magnet tripping out, the proton beam missing the target, or changes in the upstream part of our beam line (which was shared with another beam line and not always under control). The bad data were edited by means of a scatter plot which, for a given scaler, plotted for each beam pulse the (scaler/monitor) ratio versus the average instantaneous counting rate ${ }^{*}$ during that beam pulse. Separate scatter plots were made for target in and target out. Fig. 5 shows a typical plot. It was found that it was only necessary to plot one of the scaler channels for editing purposes. Plots from each run were inspected. The bad records were well separated from the good ones on the plots and were deleted from the run. After the data were edited, the observed variations in scaler/monitor ratios were generally consistent with those expected from statistics.

\subsection{Statistical errors}

Cross sections for a given run and scaler channel were calculated from eq. (1)

* This was determined by monitoring the accidental coincidence defined in eq. (18). 
with $\Sigma S_{i j}$ and $\Sigma M$ being the total counts accumulated during the run (after editing). The expected statistical error in the cross section is given by

$$
\Delta \sigma_{i j}^{\mathrm{s}}=\frac{1}{n x}\left[\left(\frac{1}{\sum S_{i j}}+\frac{1}{\sum M}\right)_{\text {out }}+\left(\frac{1}{\sum S_{i j}}+\frac{1}{\sum M}\right)_{\mathrm{in}}\right]^{\frac{1}{2}} .
$$

The error in the cross section could also be calculated from the pulse-to-pulse variation in the ratios $S_{i j} / M$ observed during a run. We define $\Delta(S / M)$ as the standard deviation in $S_{i j} / M$,

$$
\Delta(S / M)^{2}=\frac{1}{N-1} \sum_{l=1}^{N}\left[\left(\frac{S}{M}\right)_{l}-\frac{\sum S}{\sum M}\right]^{2},
$$

where $N$ is the number of beam pulses with a given target condition (typically $\sim 300$ ) and $\Sigma S$ and $\Sigma M$ are the total counts accumulated during the run. The standard deviation in $R \equiv \Sigma S / \Sigma M$ is

$$
\delta R=\overline{\Delta(S / M)} / \sqrt{N} .
$$

The "deviation error" in the cross section is then

$$
\Delta \sigma^{\mathrm{d}}=\frac{1}{n x}\left[\left(\frac{\delta R}{R}\right)_{\text {out }}^{2}+\left(\frac{\delta R}{R}\right)_{\text {in }}^{2}\right]^{\frac{1}{2}} .
$$

Generally the deviation errors $\Delta \sigma^{\text {d }}$ were approximately equal to the expected statistical errors $\Delta \sigma^{s}$. The mean value of $\Delta \sigma^{\mathrm{d}} / \Delta \sigma^{\mathrm{s}}$ for all the data was approximately 1.1. The error assigned to a given cross section in calculating the final average over all runs was $\Delta \sigma^{\mathrm{s}}$ or $\Delta \sigma^{\mathrm{d}}$, whichever was larger. If $\Delta \sigma^{\mathrm{d}}>2 \Delta \sigma^{\mathrm{s}}$, the data for that channel was discarded. Cross sections for each element were averaged in the usual way with the weight for a given cross section equal to the inverse of the square of the error.

\section{Corrections to the measured cross sections}

\subsection{Extrapolation of the data to zero solid angle}

The averaged partial cross sections were extrapolated to zero solid angle to obtain a value for the total cross section. For a well-defined beam and counters with sharp edges the partial cross section for a given counter and energy bin, $\sigma_{i j}$, is given by

$$
\sigma_{i j}=\sigma_{\mathrm{T}}-\int_{0}^{\Omega_{i}}\left[\left(\frac{\mathrm{d} \sigma}{\mathrm{d} \Omega}\right)_{\mathrm{el}}+\left(\frac{\mathrm{d} \sigma}{\mathrm{d} \Omega}\right)_{\mathrm{inel}}\right] \mathrm{d} \Omega,
$$




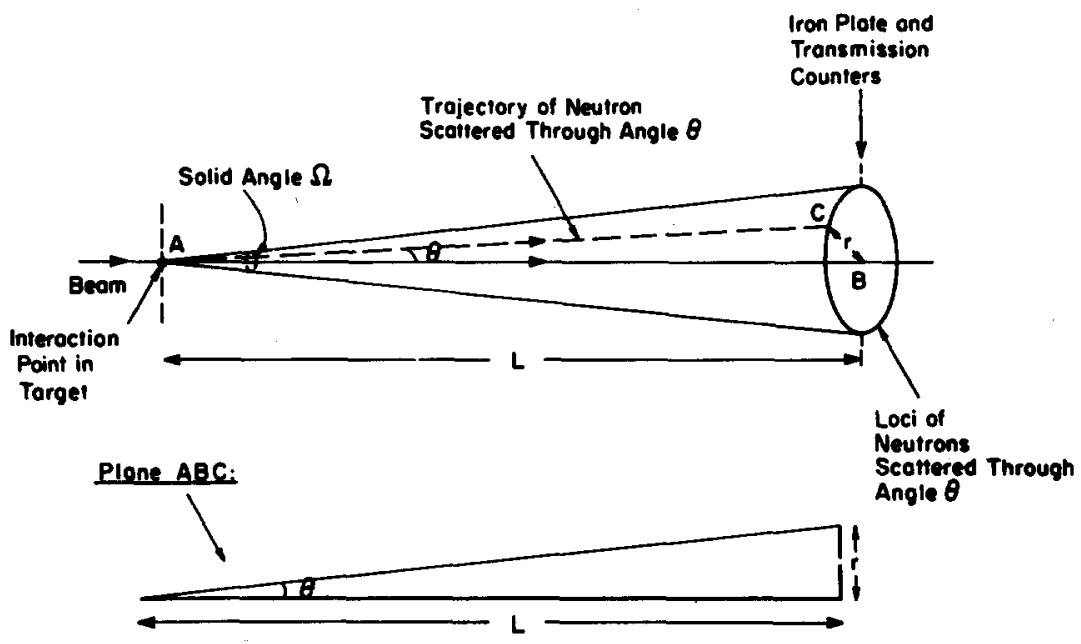

Fig. 6. Schematic diagram showing the relation between the neutron scattering angle $\theta$ and the radius $r$ at which it strikes the iron converter.

where $\sigma_{\mathrm{T}}$ is the total cross section and $\Omega_{i}$ is the solid angle subtended by the counter as seen from the target; $(\mathrm{d} \sigma / \mathrm{d} \Omega)_{\mathrm{el}}$ and $(\mathrm{d} \sigma / \mathrm{d} \Omega)_{\text {inel }}$ are the elastic and inelastic differential cross sections.

In practice however, the beam has a finite size and, more importantly, the solid angle subtended by the transmission counters is not perfectly well defined. The neutrons were required to convert in the $2.5 \mathrm{~cm}$ iron converter plate in front of the transmission counters. Because of the finite opening angle of the cone containing the charged secondaries, the effective size of the transmission counters was somewhat larger than the geometrical size. Eq. (6) must then be modified by the inclusion of an efficiency function $E_{i j}(\theta)$ for the $i$ th counter and $j$ th momentum bin which gives the probability that a neutron scattered at an angle $\theta$ from the target is detected by the counter (relative to that for unscattered neutron). The generalization of eq. (6) is

$$
\sigma_{i j}=\sigma_{\mathrm{T}}-\int_{0}^{\infty}\left[\left(\frac{\mathrm{d} \sigma}{\mathrm{d} \Omega}\right)_{\mathrm{el}}+\left(\frac{\mathrm{d} \sigma}{\mathrm{d} \Omega}\right)_{\text {inel }}\right] E_{i j}(\theta) \mathrm{d} \Omega .
$$

From fig. 6 we see that $\Omega=\pi r^{2} / L^{2}$ and $\theta=r / L$ where $L$ is the separation between the target and the transmission counters $(\approx 200 \mathrm{~m})$.

To a first approximation $(\mathrm{d} \sigma / \mathrm{d} \Omega)_{\mathrm{el}} \propto \mathrm{e}^{b t}=\mathrm{e}^{-\delta^{\prime} \theta^{2}}$ and $(\mathrm{d} \sigma / \mathrm{d} \Omega)_{\text {inel }} \simeq$ const. More generally we can expand the summed differential cross section in a polynomial in $t$ or $\theta^{2}$ as follows: 


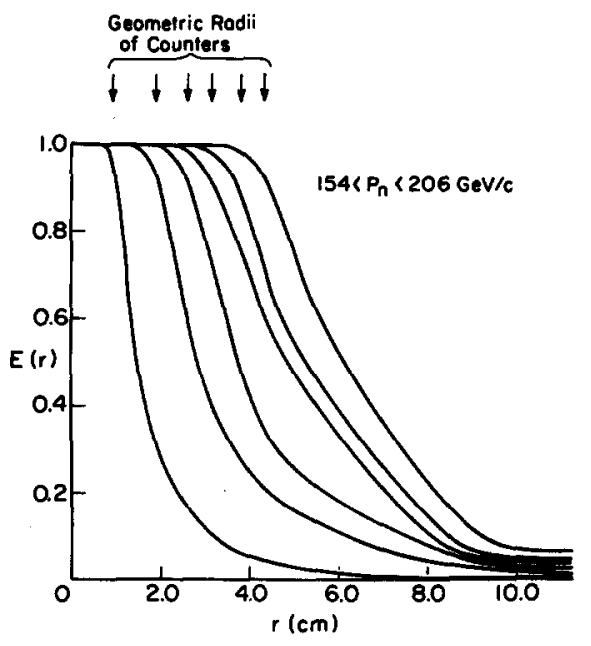

Fig. 7. Efficiency functions for the six transmission counter pairs for the momentum bin 154 to $206 \mathrm{GeV} / c$. The curves are normalized to unity at $r=0$. The geometric radii of the counters are shown.

$$
\begin{aligned}
\sigma_{i j} & =\sigma_{\mathrm{T}}-\int_{0}^{\infty}\left(\alpha+\beta \theta^{2}+\gamma \theta^{4}+\ldots\right) E_{i j}(\theta) \mathrm{d} \Omega \\
& =\sigma_{\mathrm{T}}-\alpha^{\prime} \int_{0}^{\infty} E_{i j}(r) \mathrm{d} r^{2}-\beta^{\prime} \int_{0}^{\infty} r^{2} E_{i j}(r) \mathrm{d} r^{2} \ldots
\end{aligned}
$$

We thus have a set of six linear equations (since there are 6 partial cross sections for each momentum bin). The parameters $\sigma_{\mathrm{T}}, \alpha^{\prime}, \beta^{\prime}, \gamma^{\prime}, \ldots$ can be obtained by fitting the partial cross sections. The integrals $\int r^{n} E_{i j}(r) \mathrm{d} r^{2}$ are moments of the efficiency functions which can be calculated by numerical integration using the $E_{i j}(r)$ measured as described below.

Values of the efficiency function $E_{i j}(r)$ were obtained for each pair of transmission counters and momentum bin by measuring the counting rate (with no target in the beam) when the converter plate and transmission counters were moved a distance $r$ from their normal position. The ratio $S_{i j} / M$ normalized to its value at $r=0$ is then $E_{i j}$.

Such scans were made several times during the course of the experiment. Both horizontal and vertical scans were made. These were found to differ slightly so an average of the two was used for $E_{i j}$. Fig. 7 shows a typical set of curves for $E_{i j}$. Forty two such curves were obtained. Values were read off each curve for use in the numerical integration. The geometric radii of the transmission counters are also indicated in fig. 7. As might be expected, there is a significant probability of a neutron 
Table 3

Corrections added to the cross sections measured with the smallest transmission counter to obtain the total cross section; approximate values of the total cross sections are also listed for comparison

\begin{tabular}{|c|c|c|c|c|c|c|c|c|c|}
\hline \multirow[b]{2}{*}{ Nucleus } & \multirow{2}{*}{$\begin{array}{l}\text { Atomic } \\
\text { Weight }\end{array}$} & \multirow{2}{*}{$\underset{(\mathrm{mb})}{\sigma_{T}}$} & \multirow[b]{2}{*}{34} & \multicolumn{3}{|c|}{ Momentum $(\mathrm{GeV} / \mathrm{c})$} & \multirow[b]{2}{*}{215} & \multirow[b]{2}{*}{240} & \multirow[b]{2}{*}{273} \\
\hline & & & & 80 & 131 & 180 & & & \\
\hline H & $\mathbf{1}$ & 40 & $\begin{array}{r}0.37 \\
\pm 0.15\end{array}$ & $\begin{array}{r}0.11 \\
\pm 0.06\end{array}$ & $\begin{array}{r}0.09 \\
\pm 0.05\end{array}$ & $\begin{array}{r}0.07 \\
\pm 0.04\end{array}$ & $\begin{array}{r}0.08 \\
\pm 0.04\end{array}$ & $\begin{array}{r}0.08 \\
\pm 0.04\end{array}$ & $\begin{array}{r}0.12 \\
\pm 0.06\end{array}$ \\
\hline D & 2 & 74 & $\begin{array}{r}0.65 \\
\pm 0.10\end{array}$ & $\begin{array}{r}0.20 \\
\pm 0.05\end{array}$ & $\begin{array}{r}0.20 \\
\pm 0.05\end{array}$ & $\begin{array}{r}0.23 \\
\pm 0.05\end{array}$ & $\begin{array}{r}0.25 \\
\pm 0.08\end{array}$ & $\begin{array}{r}0.30 \\
\pm 0.08\end{array}$ & $\begin{array}{r}0.30 \\
\pm 0.05\end{array}$ \\
\hline Be & 9 & 270 & $\begin{array}{r}3.7 \\
+0.6\end{array}$ & $\begin{array}{r}1.5 \\
\pm 0.2\end{array}$ & $\begin{array}{r}1.5 \\
\pm 0.2\end{array}$ & $\begin{array}{r}2.8 \\
\pm 0.3\end{array}$ & $\begin{array}{r}3.1 \\
\pm 0.5\end{array}$ & $\begin{array}{r}2.6 \\
\pm 0.4\end{array}$ & $\begin{array}{r}2.4 \\
\pm 0.6\end{array}$ \\
\hline c & 12 & 330 & $\begin{array}{r}8.4 \\
\pm 5.3\end{array}$ & $\begin{array}{r}3.4 \\
\pm 0.5\end{array}$ & $\begin{array}{r}2.5 \\
\pm 0.4\end{array}$ & $\begin{array}{r}2.4 \\
\pm 0.5\end{array}$ & $\begin{array}{r}3.3 \\
\pm 0.5\end{array}$ & $\begin{array}{r}3.5 \\
\pm 0.5\end{array}$ & $\begin{array}{r}3.8 \\
\pm 0.8\end{array}$ \\
\hline $\mathbf{A 1}$ & 27.0 & 630 & $\begin{array}{l}10.7 \\
\pm 2.7\end{array}$ & $\begin{array}{r}4.7 \\
\pm 1.4\end{array}$ & $\begin{array}{r}5.4 \\
\pm 0.8\end{array}$ & $\begin{array}{r}8.6 \\
\pm 1.3\end{array}$ & $\begin{array}{l}11.7 \\
\pm 1.8\end{array}$ & $\begin{array}{l}13.3 \\
\pm 2.0\end{array}$ & $\begin{array}{l}12.9 \\
\pm 1.9\end{array}$ \\
\hline Fe & 55.8 & 1100 & $\begin{array}{r}12.7 \\
\pm 1.9\end{array}$ & $\begin{array}{l}10.6 \\
\pm 1.6\end{array}$ & $\begin{array}{l}12.2 \\
\pm 1.8\end{array}$ & $\begin{array}{l}21 \\
\pm 3\end{array}$ & $\begin{array}{l}30 \\
\pm 4\end{array}$ & $\begin{array}{l}33 \\
\pm 5\end{array}$ & $\begin{array}{l}34 \\
\pm 7\end{array}$ \\
\hline $\mathrm{Cu}$ & 63.5 & 1200 & $\begin{array}{l}15 \\
\pm 2\end{array}$ & $\begin{array}{l}12.1 \\
\pm 1.4\end{array}$ & $\begin{array}{l}19 \\
\pm 3\end{array}$ & $\begin{array}{l}28 \\
\pm 4\end{array}$ & $\begin{array}{l}40 \\
\pm 6\end{array}$ & $\begin{array}{l}42 \\
\pm 6\end{array}$ & $\begin{array}{l}44 \\
\pm 9\end{array}$ \\
\hline cd & 112.4 & 1900 & $\begin{array}{l}28 \\
\pm 4\end{array}$ & $\begin{array}{l}22 \\
\pm 3\end{array}$ & $\begin{array}{l}42 \\
\pm 6\end{array}$ & $\begin{array}{l}58 \\
\pm 9\end{array}$ & $\begin{array}{r}84 \\
\pm 13\end{array}$ & $\begin{array}{r}89 \\
\pm 13\end{array}$ & $\begin{array}{r}88 \\
\pm 14\end{array}$ \\
\hline $\mathbf{w}$ & 183.8 & 2800 & $\begin{array}{r}44 \\
\pm 10\end{array}$ & $\begin{array}{l}33 \\
\pm 5\end{array}$ & $\begin{array}{r}84 \\
\pm 13\end{array}$ & $\begin{array}{l}116 \\
\pm 17\end{array}$ & $\begin{array}{l}158 \\
\pm 28\end{array}$ & $\begin{array}{l}162 \\
\pm 27\end{array}$ & $\begin{array}{l}178 \\
\pm 27\end{array}$ \\
\hline $\mathrm{Pb}$ & 207.2 & 3000 & $\begin{array}{l}117 \\
\pm 29\end{array}$ & $\begin{array}{l}53 \\
\pm 8\end{array}$ & $\begin{array}{r}94 \\
\pm 14\end{array}$ & $\begin{array}{l}135 \\
\pm 24\end{array}$ & $\begin{array}{l}181 \\
\pm 27\end{array}$ & $\begin{array}{l}182 \\
\pm 27\end{array}$ & $\begin{array}{l}190 \\
\pm 45\end{array}$ \\
\hline U & 238 & 3400 & $\begin{array}{l}149 \\
\pm 22\end{array}$ & $\begin{array}{r}66 \\
\pm 10\end{array}$ & $\begin{array}{l}122 \\
\pm 18\end{array}$ & $\begin{array}{l}183 \\
\pm 28\end{array}$ & $\begin{array}{l}230 \\
\pm 34\end{array}$ & $\begin{array}{l}238 \\
\pm 40\end{array}$ & $\begin{array}{l}228 \\
\pm 55\end{array}$ \\
\hline
\end{tabular}

being detected even though it was outside the geometric radius of a counter so that the effective sizes of the transmission counters were somewhat larger than their geometric sizes.

The polynomial expansion of $d \sigma / d \Omega$ leading to eq. (8) is especially convenient because it allows the use of a linear least-squares fitting technique to obtain $\sigma_{\mathrm{T}}$. The integrals $\int r^{n} E_{i j} \mathrm{~d} r^{2}$ were calculated numerically and fed into the program. For low momenta and smaller nuclei good fits were obtained with only one or two terms in the expansion. More terms were required at higher momenta but fits with 4 parameters $\left(\sigma_{\mathrm{T}}, \alpha^{\prime}, \beta^{\prime}\right.$, and $\left.\gamma^{\prime}\right)$ gave good fits even with uranium at the highest momentum. As a check, various other forms, including exponentials, were tried for $\mathrm{d} \sigma / \mathrm{d} \Omega$.

These are described below.

Table 3 gives the corrections to the cross sections measured with the smallest transmission counters along with the assigned errors. Approximate total cross sections are also included for comparison. It can be seen that these corrections range from $\approx 0.2 \%$ for hydrogen at momenta near $100 \mathrm{GeV} / c$ to $\approx 7 \%$ for uranium at the 
highest momentum. Generally the corrections rise with increasing momentum since the transmission counters subtended a fixed solid angle and thus a larger four-momentum transfer at higher neutron momenta. The corrections rise again at very low momenta. This is because high-energy neutrons which scatter inelastically feed down into the lower energy bins. This effect would disappear in the limit of an ideal point detector, so that it is automatically taken care of in the extrapolation to zero solid angle.

In assigning errors to these corrections, we took into account the following:

(i) Possible uncertainties in the efficiency function $E_{i j}$. These were measured to an accuracy of several percent at each point. However, it was assumed that a systematic error $\sim 15 \%$ was possible.

(ii) Statistical uncertainties in the measured partial cros: sections. These were generally quite small because the partial cross sections for a given momentum bin are strongly correlated.

(iii) Possible deficiencies in the fitting function. In all cases the polynomial expansion given by eq. ( 8 ) gave good fits to the partial cross sections if enough terms were included. For cadmium, lead, and uranium in the two highest momentum bins, it was found that the fitted values of $\sigma_{\mathrm{T}}$ tended to increase significantly $(\approx 4 \%)$ even though the fits did not improve significantly when the number of fitted parameters went from 4 to 5 . To investigate this question and the sensitivity of the fitted total cross sections to the form used in the fit, several other functions were also tried. The differential elastic cross section for nuclei should be well approximated by the form

$$
\left(\frac{\mathrm{d} \sigma}{\mathrm{d} t}\right)_{\mathrm{el}} \propto \mathrm{e}^{b t} .
$$

The exponential slope $b$ is related to the nuclear radius and therefore to the atomic weight so that

$$
b \simeq 10.8 A^{\frac{2}{3}} \mathrm{GeV}^{-2} \text {. }
$$

This gives $b=252,380$ and 415 for cadmium, lead, and uranium respectively. The differential inelastic scattering cross section should have an exponential slope $\sim 10 \mathrm{GeV}^{-2}$, characteristic of the nucleon-nucleon interaction. Fits were therefore tried with the form

$$
\left(\frac{\mathrm{d} \sigma}{\mathrm{d} t}\right)_{\text {total }}=a \mathrm{e}^{b t}+c \mathrm{e}^{d t},
$$

or

$$
\left(\frac{\mathrm{d} \sigma}{\mathrm{d} \Omega}\right)_{\text {total }}=\alpha \mathrm{e}^{-\beta \theta^{2}}+\gamma \mathrm{e}^{-\delta \theta^{2}},
$$

with $\alpha, \gamma$, and $\delta$ as fitted parameters and $\beta$ as predicted from eq. (10) and the relation 


$$
-t=\bar{p}^{2} \theta^{2}=\bar{p}^{2} r^{2} / L^{2},
$$

where $\bar{p}$ is the average momentum for the bin.

Fits using eq. (11) had to be done by searching for the minimum $\chi^{2}$ as the parameters $\sigma_{\mathrm{T}}, \alpha, \gamma$, and $\delta$ were varied. For each trial value of these parameters the integrations over the $E_{i j}(r)$ had to be done numerically. These fits therefore required considerably more computer time than the linear form which results from the polynomial expansion, eq. (8). These fits were found to give a $\chi^{2}$ comparable to the polynomial fits with total cross sections intermediate between those obtained with the 4 and 5-term polynomial fits. If $\beta$ was allowed to vary as a free parameter, the fits did not improve and the resulting value of $\beta$ were consistent with the value predicted from eq. (10).

We also tried fits with

$$
\left(\frac{\mathrm{d} \sigma}{\mathrm{d} \Omega}\right)_{\text {total }}=\alpha \mathrm{e}^{-\beta \theta^{2}}+\gamma \mathrm{e}^{-\delta \theta^{2}}+\epsilon \mathrm{e}^{-\xi \theta^{2}},
$$

with $\beta$ fixed at the expected value from eq. (10) and $\delta$ and $\xi$ corresponding to exponential slopes $b=10$ and $5 \mathrm{GeV}^{-2} ; \sigma_{\mathrm{T}}, \alpha, \gamma$, and $\epsilon$ were free parameters. This gave results similar to the fit using eq. (11). Other forms were also tried for $\mathrm{d} \sigma / \mathrm{d} \Omega$.

The spread between the total cross sections obtained with the various fitting functions was used to estimate the error in the extrapolation to zero solid angle. The errors in the total cross sections for the heavy nuclei in the higher momentum bins are completely dominated by the uncertainty in the extrapolation to zero solid angle.

\section{2. $K^{\circ}$ contamination}

The neutral beam contained a significant fraction of neutral kaons at low momenta. This was studied by measuring the transmission of the beam for carbon targets of various thicknesses. Since the $\mathrm{K}^{\circ}$-carbon cross section is approximately half that for neutrons, it is possible to obtain the fraction of kaons by fitting the transmission data to the sum of two exponentials in the target thickness.

With no target in the beam, we can write the total flux, $F_{0}$, in a given momentum range as

$$
F_{0}=K+N \text {, }
$$

where $K$ and $N$ are the fluxes of kaons and neutrons in the beam. If a target of length $n x$ is inserted in the beam, then the kaons and neutrons are attenuated according to their respective total cross sections and the measured flux $F(x)$ is given by

$$
F(x)=K \mathrm{e}^{-\sigma_{\mathrm{K}} n x}+N \mathrm{e}^{-\sigma_{\mathrm{n}} n x},
$$




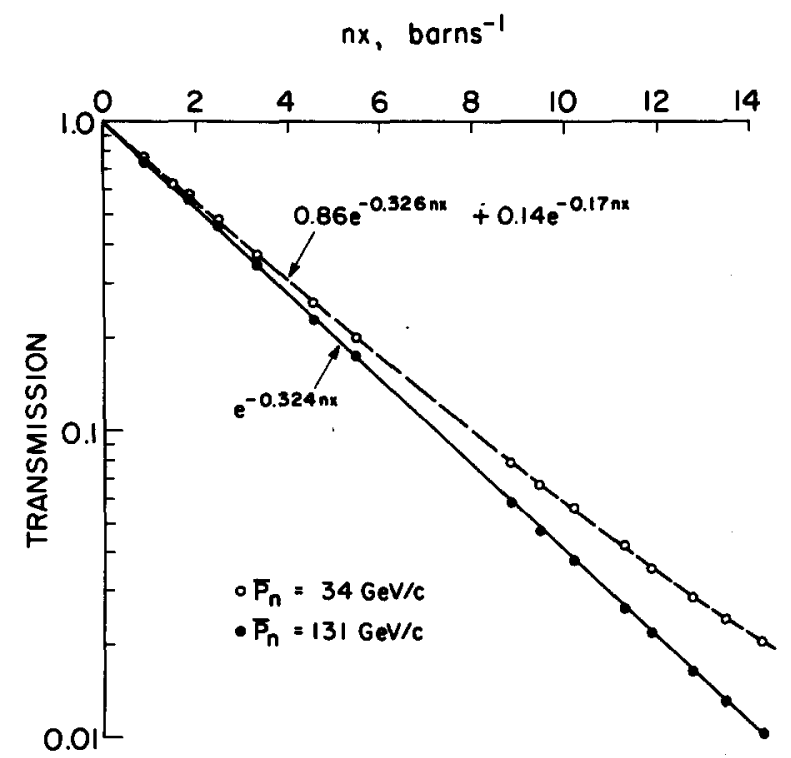

Fig. 8. Transmission of the beam versus carbon target thickness for 2 momentum ranges. Fits to the data are also plotted.

where $\sigma_{\mathrm{K}}$ and $\sigma_{\mathrm{n}}$ are the cross sections for kaons and neutrons respectively in carbon. Hence we have

$$
\begin{aligned}
T(x) & \equiv \frac{F(x)}{F_{0}}=\frac{K}{K+N} \mathrm{e}^{-\sigma_{\mathrm{K}} n x}+\frac{N}{K+N} \mathrm{e}^{-\sigma_{\mathrm{n}} n x} \\
& =f_{\mathrm{K}} \mathrm{e}^{-\sigma_{\mathrm{K}} n x}+\left(1-f_{\mathrm{K}}\right) \mathrm{e}^{-\sigma_{\mathrm{n}} n x},
\end{aligned}
$$

where $T(x)$ is the measured transmission and $f_{\mathrm{K}}$ is the apparent fraction of kaons in the beam (i.e. the fraction which converts in the iron plate and is detected). In practice $T(x)$ is a function of the solid angle subtended by the transmission counters. It was therefore extrapolated to zero solid angle (a correction $\sim 1 \%$ ) so that the cross sections in eq. (14) are total cross sections. By definition the measured cross section is

$$
\sigma_{\mathrm{m}}=\frac{-1}{n x} \ln T(x) .
$$

Graphs showing the variation of the transmission with carbon thickness for two momentum ranges are shown in fig. 8. Also plotted are fits to eq. (14) in which $f_{\mathrm{K}}$, $\sigma_{\mathrm{K}}$, and $\sigma_{\mathfrak{n}}$ were fitted parameters whose values were determined by minimizing $\chi^{2}$. 
Table 4

Effective fractions of kaons, photons, and antineutrons in the beam for each momentum bin

\begin{tabular}{|c|c|c|c|}
\hline Mean Mamentum (GeV/c) & Fraction of Kaons & Fraction of Photons & Praction of $\bar{n} \cdot s$ \\
\hline 34 & $0.138 \pm 0.038$ & $0.027 \pm 0.008$ & $0.070 \pm 0.040$ \\
80 & $0.066 \pm 0.018$ & 0.0 & 0.0 \\
131 & $0.013 \pm 0.004$ & 0.0 & 0.0 \\
180 and above & 0.0 & 0.0 & 0.0 \\
\hline
\end{tabular}

The values obtained for the apparent fraction $f_{\mathrm{K}}$ of kaons in the beam for various energy ranges are given in table 4 which also lists the fractions of photons and antineutrons in the beam * . (The determination of the photon and antineutron contamination are discussed in subsects. 4.3 and 4.4 respectively.)

Corrections to the cross section for nuclei other than carbon can be obtained as follows. From eqs. (14) and (15)

$$
\begin{aligned}
\mathrm{e}^{-\sigma_{\mathrm{m}} n x} & =f_{\mathrm{K}} \mathrm{e}^{-\sigma_{\mathrm{K}} n x}+\left(1-f_{\mathrm{K}}\right) \mathrm{e}^{-\sigma_{\mathrm{n}} n x} \\
& =\mathrm{e}^{-\sigma_{\mathrm{n}} n x}\left[1+f_{\mathrm{K}}\left(\mathrm{e}^{\left(\sigma_{\mathrm{n}}-\sigma_{\mathrm{K}}\right) n x}-1\right)\right] .
\end{aligned}
$$

Taking the logarithm and solving for $\sigma_{\mathrm{n}}$, we obtain

$$
\sigma_{\mathrm{n}}=\sigma_{\mathrm{m}}+\frac{1}{n x} \ln \left[1+f_{\mathrm{K}}\left(\mathrm{e}^{\left(\sigma_{\mathrm{n}}-\sigma_{\mathrm{K}}\right) n x}-1\right)\right] .
$$

Eq. (16) gives the corrected cross section $\sigma_{\mathrm{n}}$ for neutrons in terms of the measured cross section $\sigma_{\mathrm{m}}$, the fraction of $\mathrm{K}^{\prime} \mathrm{s}$ in the beam, and the ratio of the $\mathrm{K}^{\circ}$-nucleus and n-nucleus total cross section $\sigma_{\mathrm{K}} / \sigma_{\mathrm{n}}$. The latter were determined with sufficient accuracy from available experimental data at lower energies $[6,14]$ and theoretical predictions (see subsect. 6.3).

\subsection{Correction for contamination by photons}

A small contamination of photons was present in the beam at low energies. Corrections were made using eq. (16) with $\sigma_{\mathrm{K}}$ replaced by $\sigma_{\gamma}$ defined by [15]

$$
\sigma_{\gamma}=\frac{7}{9} \frac{A}{L_{\mathrm{R}}}
$$

* More detailed data on the kaon and photon spectra in the M3 beam can be found in an internal report [13]. 
where $A$ is the atomic weight and $L_{\mathrm{R}}$ the radiation length in $\mathrm{gm} / \mathrm{cm}^{2}$. The fraction of $\gamma$ 's in the beam for each momentum bin was determined by measuring the transmission of the beam for lead targets of various thicknesses.

\subsection{Correction for contamination by antineutrons}

At Fermilab energies the $\overline{\mathrm{n}}$-nucleus total cross sections are expected to differ by less than $10 \%$ from the $n$-nucleus cross section. It is therefore not practical to use the technique by which we determined the $K^{\circ}$ contamination for $\bar{n}$ 's. The fraction of $\bar{n}$ 's in the beam was therefore estimated from the $\bar{p} / p$ ratio in a similar beam. The $\overline{\mathbf{n}}$ contamination is negligible except for our lowest momentum bin centered near $35 \mathrm{GeV} / c$. Baker et al. [16] have measured $\overline{\mathrm{p}}$ and $\mathrm{p}$ yields in a similar charged beam line in the Meson Area. They found that the ratio of antiprotons to protons at $35 \mathrm{GeV} / c$ was $11 \%$. We have assumed that the $\bar{n} / \mathrm{n}$ ratio in the $\mathrm{M} 3$ beam line is the same as the $\bar{p} / \mathrm{p}$ ratio in the $\mathrm{M} 1$ line. Due to the questionable nature of this assumption a large uncertainty was assigned to the fraction of $\bar{n}$ 's (table 4 ). The $\bar{n}$-nucleus total cross sections were estimated from Glauber theory (see subsect. 6.3) using known $\bar{p}$ p total cross sections.

\subsection{Rate effects}

Because we made cuts on the neutron pulse height from the calorimeter, any shift in the pulse-height spectrum between target in and target out would cause a systematic error in the cross sections. Counting rates in the calorimeter were unavoidable higher $(\sim 20 \%)$ when the target was out. As a result there was a slight dependence of the measured cross sections on instantaneous counting rate (averaged over a given run). This was due to "pileup" of pulses from the calorimeter *. The situation can be understood qualitatively by referring to fig. 9 . In total cross section measurements with a charged beam the pulse height from the detector (usually a scintillation counter) is relatively well-defined and small changes in pulse height have little or no effect on the counting rate (fig. 9a). In this experiment (fig. 9b), pulseheight cuts were made on a continuous spectrum. Any shift in the spectrum between target in and out (such as that shown by the dashed curve) will cause a systematic error in the cross section. The sign and magnitude of this error is determined by the slope of the spectrum at the point where the pulse-height cuts are made. For a spectrum like that shown (which resembles ours) cross sections in the low-energy bins are slightly decreased. That for the bin straddling the peak in the spectrum will be hardly affected and that for the highest bin will be significantly increased. As shown below, these systematic shifts in the measured cross sections are (approximately) linear functions of the instantaneous counting rate (averaged over a run).

* This is an accidental coincidence between two pulses from the calorimeter which gives a pulse height larger than either alone. 


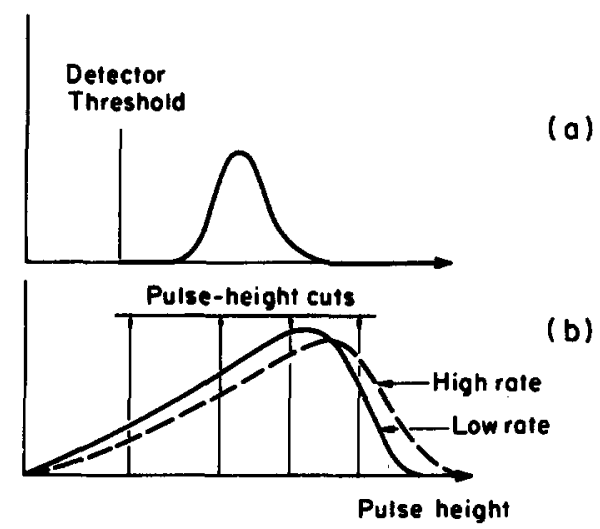

Fig. 9. Pulse-height spectrum from detector (schematic) for total cross section measurement (a) with charged beam, (b) with neutron beam with energy spectrum similar to ours.

In this experiment the effects of pileup are aggravated for two reasons:

(i) The relatively poor duty cycle of the accelerator. This was especially true in the early stages of the experiment. Over the course of the experiment the duty factor within the beam spill increased from $\approx 10 \%$ to $\approx 60 \%$. Thus we were able to study these effects over a wide range of conditions.

(ii) Only approximately $15 \%$ of the incident neutrons interacted in the converter ahead of the calorimeter and were detected in the transmission counters. The remainder still deposited their energy in the calorimeter, so that there was effectively a large background of extraneous neutrons in the calorimeter.

As mentioned previously, pileup is due to an accidental coincidence between two unrelated pulses from the calorimeter and is therefore a type of twofold accidental. During the experiment various twofold accidental rates were continuously monitored. An especially useful one was the "calorimeter accidental rate" defined as

$$
a \equiv \frac{100(\mathrm{C} \cdot \mathrm{C})}{\mathrm{C}},
$$

where $(C \cdot C)$ represents an accidental coincidence between the calorimeter output and the calorimeter output delayed $190 \mathrm{~ns}$ or 10 synchrotron rf periods. The fractional error in the (scaler/monitor) ratio due to pileup is therefore proportional to $a$, or

$$
\frac{R^{\mathrm{M}}-R^{\mathrm{T}}}{R^{\mathrm{T}}}=\gamma a,
$$

where $R^{\mathrm{M}}$ is the measured (scaler/monitor) ratio, $R^{\mathrm{T}}$ is the true value, and $\gamma$ is a proportionality constant. The measured transmission is then 
Table 5

Coefficients and $\chi^{2}$ for fits of the hydrogen cross sections to polynomials in the (target out target in) rate difference $\left(a_{\mathrm{O}}-a_{\mathrm{I}}\right)$

\begin{tabular}{|c|c|c|c|c|c|c|c|}
\hline \multirow[b]{2}{*}{$\begin{array}{c}\text { Mean Momentum } \\
(G e V / c)\end{array}$} & \multicolumn{3}{|c|}{$\begin{array}{l}\text { Fit to: } \\
\sigma=\varepsilon_{0}+\varepsilon_{1}\left(Q_{0}-Q_{1}\right)\end{array}$} & \multicolumn{4}{|c|}{$\begin{array}{l}\text { Fit to: } \\
\sigma=\varepsilon_{0}+\varepsilon_{1}\left(Q_{0}-Q_{1}\right)+\varepsilon_{2}\left(a_{0}-a_{I}\right)^{2}\end{array}$} \\
\hline & $\varepsilon_{0}$ & $\varepsilon_{1}$ & $x^{2(a)}$ & $\varepsilon_{0}$ & $\varepsilon_{1}$ & $\varepsilon_{2}$ & $x^{2(b)}$ \\
\hline 273 & $40.20 \pm 0.22$ & $4.41 \pm 0.32$ & 84.35 & $40.02 \pm 0.37$ & $4.69 \pm 1.30$ & $-0.17 \pm 0.75$ & 84.30 \\
\hline 240 & $39.58 \pm 0.23$ & $-0.02 \pm 0.30$ & 68.74 & $39.54 \pm 0.33$ & $0.17 \pm 1.15$ & $-0.12 \pm 0.67$ & 68.71 \\
\hline 215 & $39.71 \pm 0.23$ & $-0.87 \pm 0.27$ & 61.55 & $39.34 \pm 0.34$ & $0.89 \pm 1.23$ & $-1.03 \pm 0.70$ & 59.39 \\
\hline 180 & $39.55 \pm 0.19$ & $-1.19 \pm 0.25$ & 57.07 & $39.26 \pm 0.28$ & $0.16 \pm 0.98$ & $-0.81 \pm 0.57$ & 55.02 \\
\hline 131 & $38.78 \pm 0.22$ & $-1.00 \pm 0.25$ & 82.43 & $38.54 \pm 0.31$ & $0.14 \pm 1.12$ & $-0.68 \pm 0.65$ & 81.34 \\
\hline 80 & $37.56 \pm 0.25$ & $-1.19 \pm 0.27$ & 65.69 & $37.46 \pm 0.36$ & $-0.72 \pm 1.27$ & $-0.28 \pm 0.73$ & 65.55 \\
\hline 34 & $34.52 \pm 0.38$ & $-0.79 \pm 0.41$ & 77.43 & $34.44 \pm 0.54$ & $-0.32 \pm 1.90$ & $-0.28 \pm 1.09$ & 77.36 \\
\hline
\end{tabular}

(a) 78 degrees of freedom

(b) 77 degrees of freedion

$$
T^{\mathrm{M}} \equiv \frac{R_{\mathrm{I}}^{\mathrm{M}}}{R_{\mathrm{O}}^{\mathrm{M}}}=\frac{R_{\mathrm{I}}^{\mathrm{T}}\left(1+\gamma a_{\mathrm{I}}\right)}{R_{\mathrm{O}}^{\mathrm{T}}\left(1+\gamma a_{\mathrm{O}}\right)},
$$

where subscripts I and $O$ refer to target in and out respectively. Combining eq. (20) with eq. (1) and expanding the exponential we find

$$
\sigma^{\mathrm{M}} \simeq \sigma^{\mathrm{T}}+\frac{\gamma}{n x}\left(a_{\mathrm{O}}-a_{\mathrm{I}}\right)
$$

where $\sigma^{\mathrm{M}}$ and $\sigma^{\mathrm{T}}$ are the measured and true cross sections. The measured cross sections are therefore a linear function of the difference between accidental rates with target out and target in. The true cross section can be obtained by extrapolating $\sigma^{\mathrm{M}}$ to $\left(a_{\mathrm{O}}-a_{\mathrm{I}}\right)=0$.

During the experiment, cross sections for hydrogen were measured over a wide range of beam intensities (and therefore accidental rates). Fits of the hydrogen cross sections to eq. (21) could therefore be made. Linear and quadratic fits were tried. The values of the coefficients obtained and $\chi^{2}$ values are listed in table 5. It can be seen that the two fits are equally good. The linear fit was therefore used. The total cross section for hydrogen, corrected for rate effects but not kaon contamination, is $\epsilon_{0}$; the values obtained in the two fits are in good agreement.

From eq. (21) we see that the slope of the graph of $\sigma^{\mathrm{M}}$ against $\left(a_{\mathrm{O}}-a_{\mathrm{I}}\right)$ is $\gamma / n x$. As $\gamma$ is a constant for all elements (since the calorimeter does not "know" which target is in use at a given time), the slope $\epsilon_{1}$, multiplied by the target thickness $n x$ should be a constant for all elements. However, for many elements much of 
Table 6

Values obtained for $\gamma=n x \epsilon_{1}$ with various targets (see text); for a given momentum bin $\gamma$ should be the same for all targets

\begin{tabular}{|c|c|c|c|c|c|c|c|c|}
\hline \multirow[t]{2}{*}{ Target } & $\begin{array}{l}\text { Target } \\
\text { thickne日s }\end{array}$ & \multicolumn{7}{|c|}{ Mean Momentum (GeV/c) } \\
\hline & & 34 & 80 & 131 & 180 & 215 & 240 & 273 \\
\hline Hydrogen & $8.53 \cdot \mathrm{g}-\mathrm{cm}^{-2}$ & $-4.0 \pm 2.1$ & $-6.1 \pm 1.4$ & $-5.1 \pm 1.3$ & $-6.1 \pm 1.3$ & $-4.4 \pm 1.4$ & $-0.1 \pm 1.5$ & $22.5 \pm 1.6$ \\
\hline carbon & 17.7 & $-22 \pm 28$ & $-9.0 \pm 17$ & $-5.7 \pm 14$ & $-19 \pm 13$ & $-10 \pm 15$ & $-14 \pm 16$ & $14 \pm 17$ \\
\hline carbon & $30.7 \quad "$ & $-1.4 \pm 11$ & $0.8 \pm 7.8$ & $-13.4 \pm 7.2$ & $-10 \pm 6$ & $-0.5 \pm 8.2$ & $0.9 \pm 7.2$ & $23 \pm 8$ \\
\hline \multirow[t]{3}{*}{ Aluminum } & $34.4 \quad "$ & $-9.5 \pm 4.4$ & $-6.9 \pm 2.9$ & $-1.1 \pm 2.6$ & $-6.1 \pm 2.2$ & $-7.0 \pm 2.8$ & $-1.5 \pm 2.4$ & $20 \pm 3$ \\
\hline & $\begin{array}{l}\text { Weighted } \\
\text { Average }\end{array}$ & $-5.0 \pm 1.9$ & $-6.1 \pm 1.2$ & $-4.5 \pm 1.1$ & $-6.3 \pm 1.1$ & $-4.9 \pm 1.2$ & $-0.5 \pm 1.3$ & $21.7 \pm 1.4$ \\
\hline & $\begin{array}{l}x^{2}(3 \text { Deg. } \\
\text { of freedicn) }\end{array}$ & 1.71 & 0.88 & 3.54 & 1.44 & 1.11 & 0.95 & 1.02 \\
\hline
\end{tabular}

the data was taken over periods when the rates were nearly constant and it was not possible to make useful fits. Table 6 lists the values of $\gamma$ determined from the hydrogen, carbon, and aluminum targets for which we had data over a sufficiently wide range in rates to make fits. Table 6 also lists the $\chi^{2}$ and number of degrees of freedom in each fit, as well as the weighted mean of $\gamma$ for each momentum bin. The $\chi^{2}$ for the fits are quite satisfactory, and values from the different targets are consistent. However, the hydrogen data, because many runs were made over a wide range in rates, completely dominate the weighted average. Therefore, the values of $\gamma$ obtained from the hydrogen data were used for all the rate effect corrections.

\subsection{Errors in the final cross sections}

The final value for the measured total cross section is obtained by adding to the cross section extrapolated to zero solid angle the corrections for beam contamination and rate effects. The total error is obtained by adding in quadrature the errors due to statistics, extrapolation, rate correction, and beam contamination. For hydrogen and deuterium, the uncertainty in the target density is also included. The values of the total cross sections obtained for various elements in the 7 momentum bins are listed in table 7 . Table 8 lists the partial errors due to statistics, rate corrections, and beam contamination and total errors for each element.

\section{Discussion of results}

\subsection{Hydrogen}

In fig. $10 \mathrm{a}$ the results of our measurements of the np total cross section are plot- 
Table 7

Total cross sections and errors in $\mathrm{mb}$

\begin{tabular}{|c|c|c|c|c|c|c|c|}
\hline \multirow[t]{2}{*}{ Nucleus } & \multicolumn{7}{|c|}{ Mean Momentum $(\mathrm{GeV} / \mathrm{c})$} \\
\hline & 34 & 80 & 131 & 180 & 215 & 240 & 273 \\
\hline & 38.2 & 38.98 & 39.17 & 39.52 & 39.79 & 39.66 & 40.32 \\
\hline $\mathbf{H}$ & \pm 0.9 & \pm 0.33 & \pm 0.19 & \pm 0.18 & \pm 0.24 & \pm 0.24 & \pm 0.23 \\
\hline $\mathrm{D}$ & 71.7 & 72.78 & 73.30 & 74.15 & 74.48 & 75.08 & 75.18 \\
\hline & \pm 1.9 & \pm 0.83 & \pm 0.39 & \pm 0.34 & \pm 0.36 & \pm 0.38 & \pm 0.55 \\
\hline $\mathrm{Be}$ & $\begin{array}{r}263.9 \\
\pm \quad 5.7\end{array}$ & $\begin{array}{r}269.7 \\
\pm \quad 2.8\end{array}$ & $\begin{array}{r}255.5 \\
\pm \quad 1.3\end{array}$ & $\begin{array}{r}271.1 \\
\pm 1.1\end{array}$ & $\begin{array}{r}273.5 \\
\pm 1.3\end{array}$ & $\begin{array}{r}270.8 \\
\pm 1.3\end{array}$ & $\begin{array}{r}273.8 \\
\pm 1.6\end{array}$ \\
\hline C & $\begin{array}{r}331.1 \\
\pm 8.6\end{array}$ & $\begin{array}{r}331.4 \\
\pm 3.4\end{array}$ & $\begin{array}{r}329.5 \\
\pm 1.7\end{array}$ & $\begin{array}{r}331.1 \\
\pm 1.5\end{array}$ & $\begin{array}{r}333.5 \\
\pm 1.8\end{array}$ & $\begin{array}{r}331.9 \\
\pm 1.8\end{array}$ & $\begin{array}{r}328.2 \\
\pm 2.1\end{array}$ \\
\hline Al & $\begin{array}{l}628.5 \\
\pm 13.5\end{array}$ & $\begin{array}{r}636.0 \\
\pm 6.1\end{array}$ & $\begin{array}{r}633.3 \\
\pm 3.0\end{array}$ & $\begin{array}{r}534.8 \\
\pm 2.8\end{array}$ & $\begin{array}{r}633.3 \\
\pm 3.4\end{array}$ & $\begin{array}{r}634.4 \\
\pm 3.5\end{array}$ & $\begin{array}{r}629.5 \\
\pm 3.7\end{array}$ \\
\hline $\mathrm{Fe}$ & $\begin{array}{r}1100 \\
\pm 29\end{array}$ & $\begin{array}{r}1122 \\
\pm 11\end{array}$ & $\begin{array}{r}1110 \\
\pm 7\end{array}$ & $\begin{array}{r}1110 \\
\pm 8\end{array}$ & $\begin{array}{r}1112 \\
\pm 8\end{array}$ & $\begin{array}{r}1113 \\
\pm 8\end{array}$ & $\begin{array}{r}1107 \\
\pm 10\end{array}$ \\
\hline $\mathrm{Cu}$ & $\begin{array}{r}1213 \\
\pm 30\end{array}$ & $\begin{array}{r}1239 \\
\pm 11\end{array}$ & $\begin{array}{r}1228 \\
\pm 7\end{array}$ & $\begin{array}{r}1223 \\
\pm 6\end{array}$ & $\begin{array}{r}1238 \\
\pm 9\end{array}$ & $\begin{array}{r}1231 \\
\pm 9\end{array}$ & $\begin{array}{r}1225 \\
\pm 11\end{array}$ \\
\hline cd & $\begin{array}{r}1884 \\
\pm 46\end{array}$ & $\begin{array}{r}1912 \\
\pm 16\end{array}$ & $\begin{array}{r}1890 \\
\pm 11\end{array}$ & $\begin{array}{r}1885 \\
\pm 12\end{array}$ & $\begin{array}{r}1887 \\
\pm 16\end{array}$ & $\begin{array}{r}1873 \\
\pm 16\end{array}$ & $\begin{array}{r}1882 \\
\pm 18\end{array}$ \\
\hline w & $\begin{array}{r}2840 \\
\pm 72\end{array}$ & $\begin{array}{r}2804 \\
\pm 28\end{array}$ & $\begin{array}{r}2786 \\
\pm 23\end{array}$ & $\begin{array}{r}2751 \\
\pm 24\end{array}$ & $\begin{array}{r}2746 \\
\pm 35\end{array}$ & $\begin{array}{r}2748 \\
\pm 34\end{array}$ & $\begin{array}{r}2720 \\
\pm 36\end{array}$ \\
\hline $\mathrm{Pb}$ & $\begin{array}{r}2973 \\
\pm 85\end{array}$ & $\begin{array}{r}2986 \\
\pm 25\end{array}$ & $\begin{array}{r}2981 \\
\pm 21\end{array}$ & $\begin{array}{r}2951 \\
\pm 28\end{array}$ & $\begin{array}{r}2959 \\
\pm 32\end{array}$ & $\begin{array}{r}2926 \\
\pm 32\end{array}$ & $\begin{array}{r}2919 \\
\pm 48\end{array}$ \\
\hline $\mathrm{U}$ & $\begin{array}{l}3402 \\
\pm 113\end{array}$ & $\begin{array}{r}3410 \\
\pm 29\end{array}$ & $\begin{array}{r}3399 \\
\pm 26\end{array}$ & $\begin{array}{r}3361 \\
\pm 32\end{array}$ & $\begin{array}{r}3353 \\
\pm 39\end{array}$ & $\begin{array}{r}3365 \\
\pm 46\end{array}$ & $\begin{array}{r}3297 \\
\pm 60\end{array}$ \\
\hline
\end{tabular}

ted together with other direct measurements of $\mathrm{np}$ total cross sections for momenta above $4 \mathrm{GeV} / c^{*}$ (refs. [4-7]). Some pp data are shown for comparison. Our np data join smoothly with the lower energy data. The np data show a rise of approximately $1.5 \mathrm{mb}$ between 50 and $270 \mathrm{GeV} / \mathrm{c}$, which parallels that observed in the pp data. Measurements of pp total cross sections [17-25] are shown in fig. 10b for comparison. Total errors with scale errors included are shown for all the data.

For the purposes of smoothing out the data and facilitating a comparison of the np and pp data, we have fitted each to the form

* Older, less accurate data are not included when they are superseded by more recent data. 
Table 8

Contributions to the total errors from statistics, rate corrections, and beam contamination; the errors assigned to the extrapolation to zero solid angle are given in table 3

\begin{tabular}{|c|c|c|c|c|c|c|c|c|c|c|c|c|}
\hline $\begin{array}{l}\text { Type of } \\
\text { errors }\end{array}$ & $\begin{array}{c}P \\
(G e v / C)\end{array}$ & $\mathbf{E}^{(\mathbf{a})}$ & D & Be & $c$ & $\mathbf{A 1}$ & Pe & $\mathrm{Cu}$ & cd & $\boldsymbol{u}$ & $\mathbf{P b}$ & $\mathbf{v}$ \\
\hline \multirow{7}{*}{$\begin{array}{l}\text { statia- } \\
\text { tical }\end{array}$} & 34 & $\begin{array}{l}0.38 \\
0.60(b)\end{array}$ & 0.40 & 2.0 & 2.1 & 3.0 & 11 & 9.7 & 15 & 36 & 26 & 33 \\
\hline & 80 & $\begin{array}{l}0.25 \\
0.34(b)\end{array}$ & 0.26 & 1.3 & 1.5 & 2.0 & 7.5 & 6.4 & 9.9 & 22 & 17 & 21 \\
\hline & 131 & $\begin{array}{l}0.22 \\
0.30^{(b)}\end{array}$ & 0.21 & 1.1 & 1.3 & 1.0 & 6.5 & 5.3 & 8.5 & 19 & 14 & 17 \\
\hline & 180 & $\begin{array}{l}0.19 \\
0.36^{(b)}\end{array}$ & 0.18 & 1.0 & 1.2 & 1.5 & 5.6 & 4.8 & 7.4 & 17 & 12 & 15 \\
\hline & 215 & 0.23 & 0.22 & 1.2 & 1.5 & 1.9 & 6.6 & 6.0 & 8.9 & 19 & 16 & 17 \\
\hline & 240 & 0.23 & 0.25 & 1.2 & 1.4 & 1.7 & 6.3 & 6.2 & 8.5 & 21 & 24 & 23 \\
\hline & 273 & 0.22 & 0.29 & 1.4 & 2.5 & 1.9 & 7.0 & 6.7 & 10 & 23 & 16 & 24 \\
\hline \multirow{7}{*}{ Rate } & 34 & $\begin{array}{l}0.10 \\
0.41^{(b)}\end{array}$ & 0.29 & 0.5 & 1.4 & 3.2 & 2.2 & 1.2 & 2.6 & 4.0 & 9.1 & 6.7 \\
\hline & 80 & $\begin{array}{l}0.07 \\
0.20^{(b)}\end{array}$ & 0.27 & 0.3 & 0.9 & 2.1 & 1.5 & 0.8 & 1.7 & 2.6 & 6.3 & $\begin{array}{l}4.6 \\
-\end{array}$ \\
\hline & 131 & $\begin{array}{l}0.06 \\
0.20^{(b)}\end{array}$ & 0.28 & 0.3 & 0.8 & 2.0 & 1.3 & 0.7 & 1.5 & 2.8 & 5.4 & 4.2 \\
\hline & 180 & $\begin{array}{l}0.06 \\
0.22^{(b)}\end{array}$ & 0.28 & 0.3 & 0.8 & 2.0 & 1.3 & 0.7 & 1.5 & 2.3 & 5.4 & 4.0 \\
\hline & 218 & 0.06 & 0.27 & 0.3 & 0.9 & 2.1 & 1.4 & 0.8 & 1.6 & 2.7 & 6.0 & 3.8 \\
\hline & 200 & 0.05 & 0.27 & 0.4 & 1.0 & 2.4 & 1.6 & 0.9 & 1.8 & 3.0 & 7.1 & 4.2 \\
\hline & 273 & 0.06 & 0.46 & 0.4 & 1.2 & 2.5 & 1.6 & 0.9 & 2.0 & 3.3 & 7.51 & 4.4 \\
\hline \multirow{3}{*}{$\begin{array}{l}\text { Bess } \\
\text { Contam- } \\
\text { ination (c) }\end{array}$} & 34 & $\begin{array}{l}0.81 \\
0.84^{(b)}\end{array}$ & 1.8 & 5.3 & 6.3 & 12.5 & 26 & 28 & 43 & 62 & 75 & 105 \\
\hline & 80 & $\begin{array}{l}0.37 \\
0.20^{(b)}\end{array}$ & 0.74 & 2.4 & 2.9 & 5.2 & 8.0 & 8.7 & 12 & 16 & 16 & 18 \\
\hline & 131 & $\begin{array}{l}0.08 \\
0.04^{(b)}\end{array}$ & 0.17 & 0.5 & 0.6 & 1.2 & 1.8 & 2.0 & 2.7 & 3.5 & 3.5 & 4.0 \\
\hline
\end{tabular}


Table 8 (continued)

\begin{tabular}{|c|c|c|c|c|c|c|c|c|c|c|c|c|}
\hline $\begin{array}{l}\text { Type of } \\
\text { Error: }\end{array}$ & $\frac{P}{(G e v / c)}$ & $\mathbf{B}^{(\mathbf{a})}$ & D & Be & c & A1 & Fe & $\mathrm{Cu}$ & cd & $n$ & $P B$ & $u$ \\
\hline \multirow{7}{*}{ ronel } & 34 & $0.90^{\text {(d) }}$ & 1.87 & 5.7 & 8.6 & 13.5 & 29 & 30 & 46 & 72 & 85 & 113 \\
\hline & 80 & $0.33^{(d)}$ & 0.83 & 2.8 & 3.4 & 6.1 & 11 & 11 & 16 & 28 & 25 & 29 \\
\hline & 131 & $0.19^{(d)}$ & 0.39 & 1.3 & 1.7 & 3.0 & 7 & 7 & 11 & 23 & 21 & 26 \\
\hline & 180 & $0.18^{\text {(d) }}$ & 0.34 & 1.1 & 1.5 & 2.8 & 7 & 6 & 12 & 24 & 28 & 32 \\
\hline & 215 & 0.24 & 0.36 & 1.3 & 1.8 & 3.4 & 8 & 9 & 16 & 35 & 32 & 39 \\
\hline & 240 & 0.24 & 0.38 & 1.3 & 1.8 & 3.5 & 8 & 9 & 16 & 34 & 32 & 46 \\
\hline & 273 & 0.23 & 0.55 & 1.6 & 2.1 & 3.7 & 10 & 21 & 18 & 36 & 48 & 60 \\
\hline
\end{tabular}

(a) The statistical error for the np measurement includes the error for rate correction; the rate correction errors shown are typical values.

(b) Taken with a $200 \mathrm{GeV} / c$ incident proton beam.

(c) Beam contamination errors for the highest four-momentum bins are zero.

(d) Combined errors for measurement with 200 and $300 \mathrm{GeV} / c$ incident proton beams.

$$
\sigma_{\mathrm{T}}=a_{1}\left(\ln P_{\mathrm{L}}\right)^{-2}+a_{2}\left(\ln P_{\mathrm{L}}\right)^{-1}+a_{3}+a_{4} \ln P_{\mathrm{L}}+a_{5}\left(\ln P_{\mathrm{L}}\right)^{2},
$$

where $P_{\mathrm{L}}$ is the momentum in the lab system. In these fits all the data in figs. 10a and $10 \mathrm{~b}$ were included. The np fit was forced to tie on smoothly to the pp fit at momenta $\gtrsim 400 \mathrm{GeV} / c$. The parameters for the two fits are given in table 9. As can be seen from fig. 10 , the two fits do not differ significantly above $5 \mathrm{GeV} / c$.

\subsection{Deuterium}

Our nd total cross sections are displayed in fig. 11 along with other measurements of nd and pd total cross sections. A fit to the data above $4 \mathrm{GeV} / c$ of the form in eq. (22) is also shown. As expected from charge symmetry the nd and pd cross sections are in generally good agreement. However, the data of Galbraith et al. [22] lie well below the trend of the other data. Riley [26] attributes this systematic discrepancy, which is also apparent in the higher energy pp data of Galbraith et al. (see fig. 10b), to an underestimate of the correction for the finite solid angle subtended by their detector. 


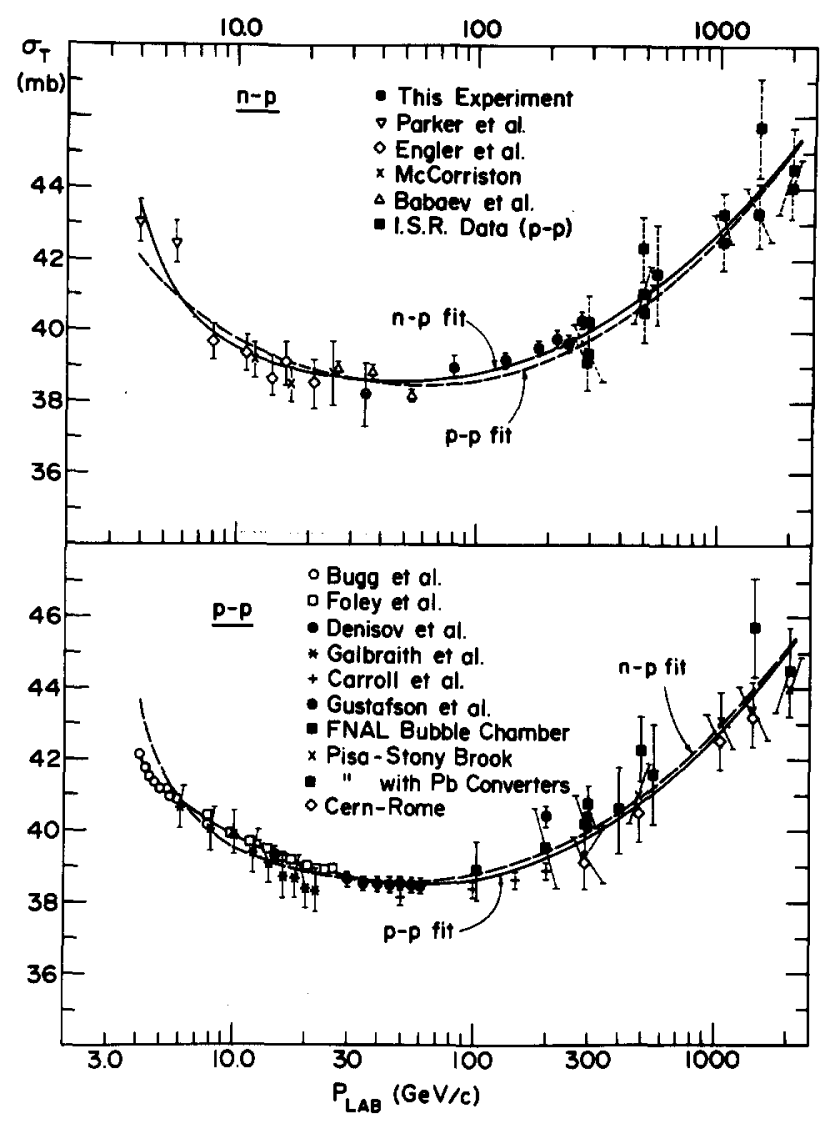

Fig. 10. (a) np total cross section from this experiment and previous measurements with neutron beams above $4 \mathrm{GeV} / c$. Some pp data are shown with dashed error bars for comparison. (b) pp total cross section data above $4 \mathrm{GeV} / c$. (Total errors, including scale errors, are shown on all points. The smooth curves are fits to eq. (22).)

\subsection{Heavy elements}

Our results for beryllium, carbon, aluminum, iron, copper, cadmium, tungsten, lead, and uranium targets are given in table $7 *$. If we fit these data at each momentum to the expression

$$
\sigma_{\mathrm{T}}(A)=\sigma_{0} A^{\nu}
$$

* A small correction $(\lesssim 0.1 \%$ ) has been made to the heavy element data for electromagnetic scattering due to the magnetic moment of the neutron (ref. [5a]). 
Table 9

Parameters for the fits of the np, pp, and Nd total cross section data to the form $\sigma_{\mathrm{T}}=a_{1}\left(\ln P_{\mathrm{L}}\right)^{-2}+a_{2}\left(\ln P_{\mathrm{L}}\right)^{-1}+\ldots+a_{5}\left(\ln P_{\mathrm{L}}\right)^{2}$

\begin{tabular}{llll}
\hline & $\sigma_{\mathrm{T}}{ }^{(\mathrm{np})}$ & \multicolumn{1}{c}{$\sigma_{\mathrm{T}}(\mathrm{pp})$} & $\sigma_{\mathrm{T}}(\mathrm{Nd})$ \\
\hline $\mathrm{a}_{1}$ & 47.267 & 16.709 & -10.165 \\
$\mathrm{a}_{2}$ & -55.832 & -23.464 & 18.901 \\
$\mathrm{a}_{3}$ & 68.257 & 57.504 & 78.248 \\
$\mathrm{a}_{4}$ & -7.395 & -6.077 & -5.094 \\
$\mathrm{a}_{5}$ & 0.685 & 0.632 & 0.706 \\
\hline
\end{tabular}

where $A$ is the atomic weight, we obtain the values for the exponent shown in fig. 12. Values obtained from lower energy data are also included. As might be expected with such a simple parametrization, the $\chi^{2}$ for these fits was often rather poor, so the errors for $\nu$ were scaled by the ratio $\left(\chi^{2} / D\right)^{\frac{1}{2}}$ where $D$ is the number of degrees of freedom. The fits show that $\nu$ has little energy dependence above $5 \mathrm{GeV} / \mathrm{c}$ This contrasts with theoretical predictions that nuclei should become significantly more opaque [27] or less opaque [28] at very high energies. There is a hint of a

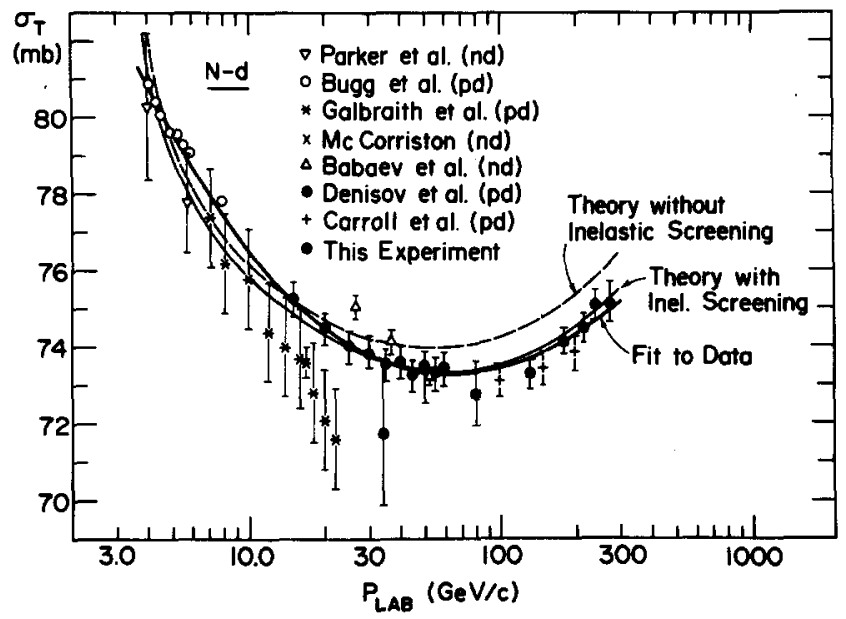

Fig. 11. Nd total cross section data above $4 \mathrm{GeV} / c$. Total errors, including scale errors, are shown on all points. The heavy solid curve is a fit to eq. (22). The light solid and dashed curves are theoretical cross sections calculated with and without the inelastic screening corrections. 


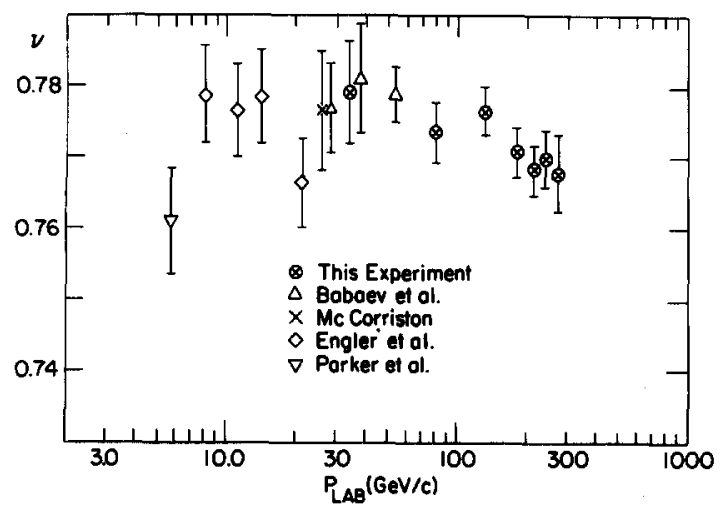

Fig. 12. Values of the exponent $v$ from fits of the heavy element total cross sections $(A \geqslant 9)$ to $\sigma_{\mathrm{T}}(A)=\sigma_{0} A^{\nu}$.

maximum in $\nu$ near $40 \mathrm{GeV} / c$. This probably reflects the minimum in the $\mathrm{NN}$ total cross sections near that momentum (fig. 10).

Total cross sections for neutrons on beryllium, carbon, aluminum, and lead are now available over a wide range of momenta. In figs. $13 \mathrm{a}$ and $13 \mathrm{~b}$ we plot our results and previous data [4-7] above $4 \mathrm{GeV} / \mathrm{c}^{*}$. Again our results join smoothly with the lower energy data. The figures also show theoretical curves which are discussed in the next section.

\section{Comparison with theory}

\subsection{Hydrogen}

The np total cross section is expected to be nearly equal to the pp at high energies. The data are in good agreement with the prediction; indeed, the two sets of data are barely distinguishable above $5 \mathrm{GeV} / c$.

Kane and Seidl [29] have calculated the difference expected between $\sigma_{\mathrm{T}}(\mathrm{np})$ and $\sigma_{\mathrm{T}}(\mathrm{pp})$ in an absorptive Regge pole model. They determine the amplitudes by fitting the data for $\mathrm{NN}$ and $\overline{\mathrm{N}} \mathrm{N}$ elastic scattering and polarization above $5 \mathrm{GeV} / c$. Their result is $\left[\sigma_{\mathrm{T}}(\mathrm{np})-\sigma_{\mathrm{T}}(\mathrm{pp})\right] \simeq+0.6 \mathrm{mb}$ at $20 \mathrm{GeV} / c$ and $+0.3 \mathrm{mb}$ at $100 \mathrm{GeV} / c$. The data (see fig. 10) are barely consistent with this large a difference at $20 \mathrm{GeV} / c$.

Our fit to the direct np total cross section (which has been forced to tie on to the

* Data from older experiments which have been superseded by more accurate results have not been included. 
pp data at very high momenta) crosses the pp fit at 6 and $35 \mathrm{GeV} / c$. Bouquet et al. [30] have noted a crossover near $40 \mathrm{GeV} / c$ if the pn total cross section data obtained by a (pd-pp) subtraction technique is compared with the pp data. This crossover, if true, would pose a significant problem in the theory [30]. However, in view of the obvious systematic effects already noted in the data, this crossover can hardly be taken seriously. The (pd-pp) measurements have the additional uncertainty in the deuteron screening correction, as discussed in sect. 1.

\subsection{Deuterium}

There has been an enormous theoretical effort devoted to the calculation of the cross section for deuterons from those for neutrons aild protons [27,31-35]. Much of this effort was motivated by the need to develop a reliable theory for extracting $\mathrm{np}$ total cross sections from experimental pd and pp total cross sections. Until the development of reliable techniques for direct measurements of $n p$ total cross sections [3-7], the (pd-pp) subtraction technique was the source of most of the np total cross section data at high energies. In terms of the nucleon-deuteron total cross section $\sigma(\mathrm{Nd})$, the np total cross section is given by

$$
\sigma(\mathrm{pn})=\sigma(\mathrm{Nd})-\sigma(\mathrm{pp})+\Delta
$$

The shadowing correction $\Delta$ must be evaluated with the assistance of a theory. At high energies $\Delta \simeq 4 \mathrm{mb}$ or approximately $10 \%$ of the np total cross section. Historically, the uncertainty in $\Delta$, as discussed in sect. 1 has been $\sim 1 \mathrm{mb}$. Thus, the uncertainty in $\Delta$ is considerably larger than the typical errors in our np total cross sections and in much of the lower energy data (fig. 10a).

We therefore choose to use the direct measurements of $\sigma(\mathrm{np}), \sigma(\mathrm{pp})$, and $\sigma(\mathrm{Nd})$ as a test of the theory. At a given energy this comparison is hampered by obvious systematic discrepancies between experiments. From fig. 10a the np experiments appear to be in generally good agreement, but there is considerable disagreement in the pp data around $20 \mathrm{GeV} / c$ and $250 \mathrm{GeV} / c$ (fig. $10 \mathrm{~b}$ ). The $\mathrm{Nd}$ data show discrepancies of about $2 \mathrm{mb}$ in the momentum range 15 to $30 \mathrm{GeV} / c$, though as previously mentioned, the data of Galbraith et al. [22] are likely to be systematically low [26]. It appears hopeless to use the data to test the theory in a limited energy range. However, a significant test is possible if we take a global view and compare all the high-energy data $\left(P_{\mathrm{lab}}>4 \mathrm{GeV} / \mathrm{c}\right)$ with the theory.

For this purpose we use the global fits to the np, pp, and Nd data shown in figs. 10 and 11. The hope is that these provide a sensible way of smoothing the data and of averaging out some of the systematic errors. In particular, it is likely that lumping the nd data and pd data together should help cancel some systematic differences between the $\mathrm{np}$ and $\mathrm{pp}$ data. 


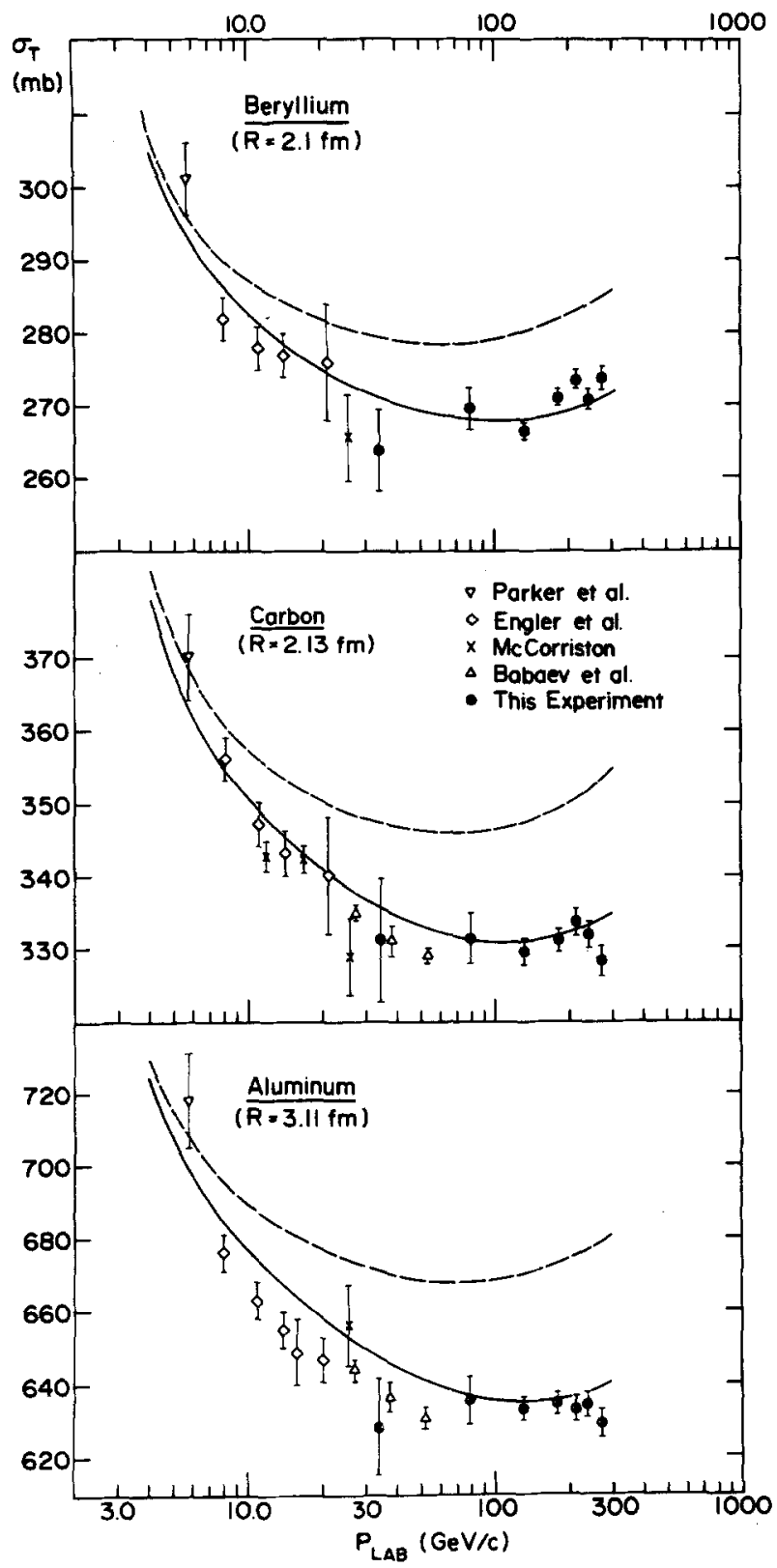

Fig. 13a. 


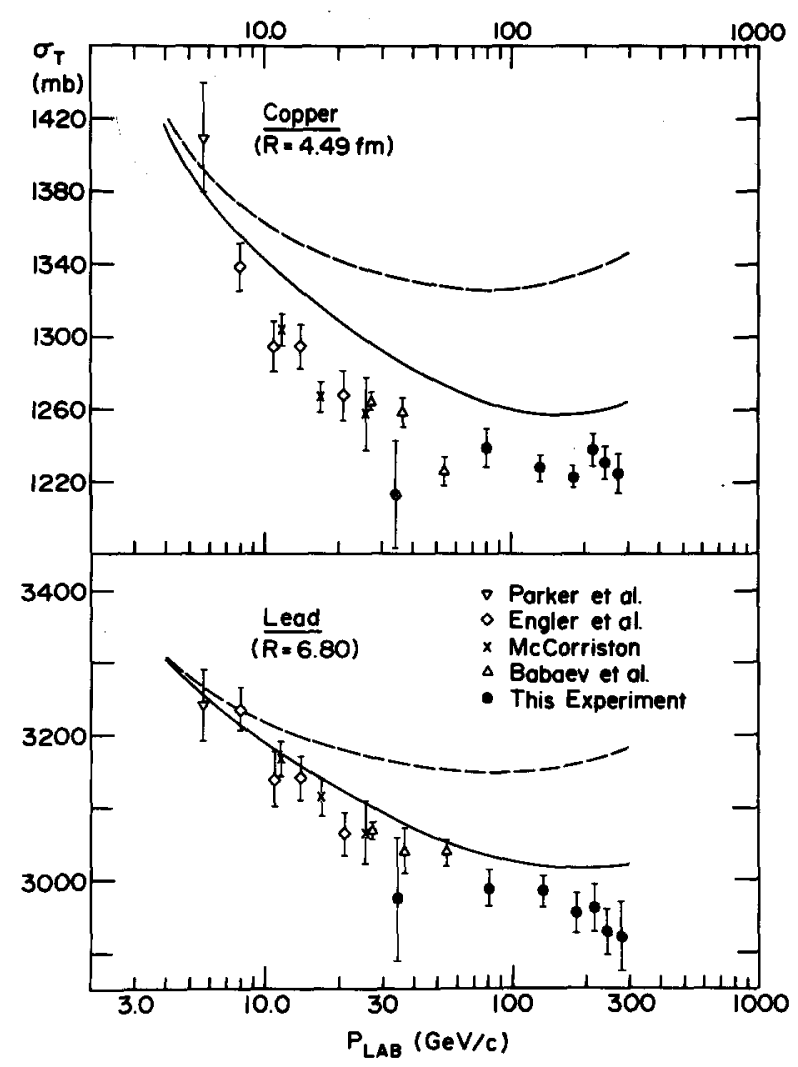

Fig. 13b.

Fig. 13. (a,b) Total cross sections for neutrons on various nuclei. The solid and dashed curves are theoretical predictions with and without inelastic screening corrections respectively; the nuclear radii were chosen to give the best agreement with the data at low momenta.

It is convenient to break the shadowing correction into two terms

$$
\Delta=\Delta_{\mathrm{el}}+\Delta_{\text {inel }} \text {. }
$$

Here $\Delta_{\mathrm{el}}$ is the well-known Glauber term which results from the diagram in fig. 14a in which the intermediate particle is a nucleon [31]; $\Delta_{\text {inel }}$, the "inelastic" screening term, is due to diagrams like that in fig. $14 \mathrm{~b}$ in which the intermediate particle is an

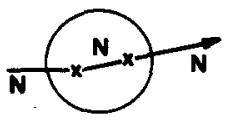

(a)

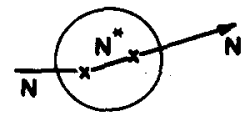

(b)

Fig. 14. (a) The double scattering diagram in conventional Glauber theory. The intermediate particle is a nucleon. (b) Double scattering diagram in which the intermediate particle is an excited state of the nucleon. The contribution from this diagram is the "inelastic screening". 
excited state of the nucleon. There has been considerable discussion concerning the existence and magnitude of this term [27,32-35]. To date there has been no firm experimental evidence for its necessity.

The elastic screening term can be written in the form [31]

$$
\Delta_{\mathrm{el}}=\frac{{ }_{\mathrm{pp}} \sigma_{\mathrm{np}}}{8 \pi^{2}}\left(1-\alpha_{\mathrm{p}} \alpha_{\mathrm{n}}\right) \int S\left(q^{2}\right) \mathrm{e}^{-\bar{\beta} q^{2}} \mathrm{~d} q
$$

where $q$ is the momentum transferred to the deuteron; $\sigma_{\mathrm{pp}}$ and $\sigma_{\mathrm{np}}$ are the total cross sections; $\alpha_{p}$ and $\alpha_{n}$ are the ratio of the real to imaginary parts of the forward pp and np scattering amplitudes; $S\left(q^{2}\right)$ is the deuteron charge form factor ${ }^{*}$, and $\bar{\beta}$ is the exponential slope for forward elastic scattering (expressed in the form $\left.\mathrm{d} \sigma / \mathrm{d} t \propto \mathrm{e}^{\beta t}\right)$ averaged between $\mathrm{np}$ and pp systems.

The values of $\sigma_{p p}$ and $\sigma_{n p}$ were taken from the global fits shown in fig. 10. In the energy range 4 to $300 \mathrm{GeV} / c, \alpha_{p}$ is fairly small and rather well known [36]. Some data on $\alpha_{n}$ are available as are dispersion relations calculations [37]. The near equality of $\sigma_{p p}$ and $\sigma_{n p}$ at high energies leads to the result that $\alpha_{n} \simeq \alpha_{p}$ at high energies [38]. Values of $\bar{\beta}$ were obtained from the data in refs. $[37,39]$. (The calculated $\Delta_{\mathrm{el}}$ are not sensitive to the choice of $\bar{\beta}$ and $\alpha$.) For $S\left(q^{2}\right)$ we have used a fit to the electron scattering data which was suggested by Anisovich et al. [34].

$$
S\left(\frac{1}{4} q^{2}\right)=0.55 \mathrm{e}^{-.19 .66 q^{2}}+0.45 \mathrm{e}^{-4.67 q^{2}} .
$$

The resulting values of $\Delta_{\mathrm{el}}$ are listed in table 10 along with the parameters used in the calculation. Other choices of $S\left(q^{2}\right)$ can give values differing as much as $10 \%$ from ours **.

For the inelastic screening term we use the relation given by Gribov [32]

$$
\Delta_{\text {inel }}=2 \int S(t) \frac{\mathrm{d}^{2} \sigma}{\mathrm{d} M^{2} \mathrm{~d} t} \mathrm{~d} M^{2} \mathrm{~d} t .
$$

Here $\mathrm{d}^{2} \sigma / \mathrm{d} M^{2} \mathrm{~d} t$ is the cross section for producing a state $\mathrm{X}$ with mass $M$ in the inclusive process $\mathrm{N}+\mathrm{N} \rightarrow \mathrm{N}+\mathrm{X}$. Eq. (28) is essentially the same as that used by Pumplin and Ross [37]. As is well known, if the low energy data (10 to $30 \mathrm{GeV})$ for $\mathrm{d}^{2} \sigma / \mathrm{d} M^{2} \mathrm{~d} t$ are used, the resulting values for the inelastic screening are too large, especially for heavy nuclei [40]. However, Kaidalov and Kondratyuk [35] argue that only the diffractive part of $\mathrm{d}^{2} \sigma / \mathrm{dM}^{2} \mathrm{~d} t$ should be included in the calculation $\dagger$. The non-diffractive background produces a very small contribution because of a phase

* We use Glauber's definition of $S\left(q^{2}\right)$. This differs slightly from the one used in refs. [33-35].

** We have also tried the empirical form given by Franco and Varma [37b]; this gave essentially the same results for the screening corrections.

$\dagger$ i.e., the part due to vacuum exchange in the $t$-channel. 
Table 10

The values of $\bar{\alpha} \equiv \frac{1}{2}\left(\alpha_{\mathrm{p}}+\alpha_{\mathrm{n}}\right), \bar{\beta}, \sigma_{\mathrm{pp}}$, and $\sigma_{\mathrm{np}}$ used in calculating $\Delta_{\mathrm{el}}$ and $\Delta_{\mathrm{inel}}$, together with the resulting Nd total cross sections

\begin{tabular}{|c|c|c|c|c|c|c|c|}
\hline $\begin{array}{c}P_{\mathrm{Lab}} \\
(\mathrm{GeV} / \mathrm{c})\end{array}$ & $\bar{\alpha}$ & $\begin{array}{c}\bar{\beta} \\
\left(\mathrm{GeV}^{2}\right)\end{array}$ & $\begin{array}{c}\sigma_{\mathrm{Pp}} \\
(\mathrm{mb})\end{array}$ & $\begin{array}{c}\sigma_{\mathrm{np}} \\
(\mathrm{mb})\end{array}$ & $\begin{array}{c}\Delta_{\mathrm{el}} \\
(\mathrm{mb})\end{array}$ & $\begin{array}{c}\Delta_{\text {inel }} \\
(\mathrm{mb})\end{array}$ & $\begin{array}{c}\sigma_{\mathrm{Na}}(\mathrm{a}) \\
(\mathrm{mb})\end{array}$ \\
\hline 4 & -0.42 & 7.5 & 42.06 & 43.64 & 3.64 & 0.11 & 81.95 \\
8 & -0.37 & 8.0 & 40.18 & 39.92 & 3.28 & 0.24 & 76.58 \\
12 & -0.31 & 9.5 & 39.57 & 39.30 & 3.20 & 0.32 & 75.35 \\
20 & -0.26 & 10.1 & 39.00 & 38.88 & 3.16 & 0.42 & 74.30 \\
30 & -0.21 & 10.6 & 38.70 & 38.70 & 3.15 & 0.49 & 73.76 \\
50 & -0.17 & 11.0 & 38.50 & 38.63 & 3.15 & 0.60 & 73.38 \\
80 & -0.12 & 11.1 & 38.55 & 38.73 & 3.20 & 0.70 & 73.38 \\
100 & -0.10 & 11.2 & 38.62 & 38.84 & 3.22 & 0.74 & 73.50 \\
150 & -0.04 & 11.6 & 38.91 & 39.15 & 3.26 & 0.83 & 73.97 \\
200 & -0.02 & 11.6 & 39.23 & 39.46 & 3.31 & 0.89 & 74.49 \\
250 & -0.01 & 11.6 & 39.53 & 39.76 & 3.34 & 0.93 & 75.02 \\
300 & -0.01 & 11.8 & 39.82 & 40.04 & 3.39 & 0.97 & 75.50 \\
\hline
\end{tabular}

(a) Calculated nucleon-deuteron total cross section with inelastic screening.

factor (neglected in eq. (28)). They isolated the diffractive part by extrapolating the date then available $\left(P_{\mathrm{lab}}<30 \mathrm{GeV} / c\right)$ to infinite energy. Since then, considerable new data have become available at Fermilab energies [41]. These show that $\mathrm{d}^{2} \sigma / \mathrm{d} M^{2} \mathrm{~d} t$ at small $t$ is approximately independent of $P_{\mathrm{lab}}$ for $P_{\mathrm{lab}} \gtrsim 50 \mathrm{GeV} / c$. In other words the high-energy limit has been achieved to a good approximation. We have therefore used the Fermilab data for $\mathrm{d}^{2} \sigma / \mathrm{d} M^{2} \mathrm{~d} t$. The detailed form used is discussed in the next section. The deuteron form factor $S(t)$ is that given in eq. (27) with $t=-q^{2}$.

The resulting values of $\Delta_{\text {inel }}$ are listed in table 10 . At $300 \mathrm{GeV} / c, \Delta_{\text {inel }} \approx 1 \mathrm{mb}$. Because of the approximations involved in eq. (28), the uncertainty in the size (and sign) of the contribution from the non-diffractive background, and the uncertainty in the normalization of the data for $\mathrm{d}^{2} \sigma / \mathrm{d} M^{2} \mathrm{~d} t$ (which is $\sim 20 \%$ ), the estimated uncertainty in $\Delta_{\text {inel }}$ is $\approx \pm 40 \%$.

The deuteron cross section calculated with and without the inelastic shadowing term are shown in fig. 11 . The theoretical curve without the inelastic screening diverges from the global fit to the data at high energies. The agreement is much better when inelastic screening is included. In view of the large uncertainties in the experimental values of $\sigma(\mathrm{Nd})$ and the calculated $\Delta_{\text {inel }}$, we conclude that the theory with inelastic screening and the data are in excellent agreement. It is worth emphasizing that there are no free parameters in the theory. 


\subsection{Heavy elements}

Total cross section data for neutrons on $\mathrm{Be}, \mathrm{C}, \mathrm{Al}, \mathrm{Cu}$, and $\mathrm{Pb}$ targets are now available over a broad energy range (figs. 13a and 13b). The data from the various experiments seem to tie together smoothly. The main difficulty in comparing the data with theory is the relatively large uncertainty in nuclear radii. However, as discussed below, this uncertainty does not affect the energy dependence of the calculated cross sections. We can therefore choose nuclear radii which give theoretical cross sections in agreement with the lower energy data and compare the energy dependence of the theory and the data. As a byproduct, we obtain values for the nuclear radii.

Franco [42] has made a detailed comparison of the low energy $(<30 \mathrm{GeV} / c)$ neutron total cross-section data with a Glauber theory calculation. At these energies, inelastic screening corrections are small, and he was unable to draw firm conclusions about their necessity.

In our calculation we use the Glauber model expression given by Franco

$$
\begin{aligned}
& \sigma_{\mathrm{T}}(\mathrm{n}-\mathrm{A})=4 \pi \operatorname{Re} \int_{0}^{\infty}\left\{1-\left[1-\frac{(1-i \bar{\alpha}) \bar{\sigma}}{4 \pi} \int_{0}^{\infty} J_{0}(q b)\right.\right. \\
& \left.\left.\times \mathrm{e}^{-\frac{1}{2} \beta q^{2}} S(q) q \mathrm{~d} q\right]^{A}\right\} b \mathrm{~d} b,
\end{aligned}
$$

where $b$ is the impact parameter, $\bar{\sigma} \equiv \frac{1}{2}\left(\sigma_{\mathrm{pp}}+\sigma_{\mathrm{np}}\right)$ and $S(q)$ is the form factor of the nucleus, which is assumed to be spherically symmetric,

$$
S(q)=\frac{4 \pi}{q} \int_{0}^{\infty} r \sin q r \rho(r) \mathrm{d} r .
$$

Franco [42] has found that the $A$ dependence of the low-energy neutron total crosssection data is reproduced best if a Woods-Saxon form is used for the nuclear density distribution. $\rho(r)$. We therefore choose the Woods-Saxon shape defined by

$$
\rho(r)=\rho_{0}\left[1+\exp \left[\frac{r-R}{(s / 4.394)}\right]\right]^{-1},
$$

where $R$ is the half-density radius and $s$ is the skin depth; $\rho_{0}$ is a normalization constant calculated from the condition $\int \rho(r) \mathrm{d} r=1$ which yields

$$
\rho_{0}=\frac{3}{4 \pi R^{3}}\left(1+0.0518 \pi^{2} s^{2} / R^{2}\right)^{-1} .
$$

Eq. (29) does not include the effects of inelastic screening. These were calculated separately as described below.

The calculation of total cross sections from eq. (29) requires the numerical evalu- 
ation of a triple integral. The integrations were done with an accuracy sufficient to insure an accuracy of at least $0.3 \%$ in the final cross section. As a check on our program, we compared our results with values calculated by Franco [42] for the same input parameters. Agreement to $<0.15 \%$ was found.

For each nucleus the total cross section was calculated for two or more values of the half-density radius $R$. A value of $R$ was then chosen to give good agreement between the calculated and measured cross sections below $10 \mathrm{GeV} / \mathrm{c}$. The skin thickness $s$ was fixed at $2.3 \mathrm{fm}$ for all nuclei. The other input parameters in the calculation $\sigma_{\mathrm{pp}}, \sigma_{\mathrm{np}}, \bar{\alpha}$, and $\bar{\beta}$ are those given in table 10 .

As mentioned previously, the effects of inelastic screening were put in separately. To evaluate them we used the expression given by Karmanov and Kondratyuk [43]:

$$
\Delta_{\text {inel }}=4 \pi \int \mathrm{d}^{2} b \int \mathrm{d} M^{2} \frac{\mathrm{d}^{2} \sigma(t=0)}{\mathrm{d} M^{2} \mathrm{~d} t} \exp \left[-\frac{1}{2} \sigma_{\mathrm{T}} T(b)\right]\left|F\left(q_{\mathrm{L}}, b\right)\right|^{2},
$$

where $b$ is the impact parameter, $\mathrm{d}^{2} \sigma(t=0) / \mathrm{d} M^{2} \mathrm{~d} t$ is the differential cross section for the process $\mathrm{N}+\mathrm{N} \rightarrow \mathrm{N}+\mathrm{X}$ evaluated at $t=0$,

$$
T(b) \equiv \int_{-\infty}^{\infty} \rho(b, z) \mathrm{d} z, \quad q_{\mathrm{L}}=\left(M^{2}-m\right) m / s
$$

is the longitudinal momentum transfer in the production of a mass $M$, and $F$ is the form factor

$$
F\left(q_{\mathrm{L}}, b\right)=\int \rho(b, z) \mathrm{e}^{i q_{\mathrm{L}} z} \mathrm{~d} z .
$$

In evaluating $\Delta_{\text {inel }}$ we have used the Woods-Saxon density $\rho(r)$ given in eq. (31) with $r=\left[b^{2}+z^{2}\right]^{\frac{1}{2}}$. The $\sigma_{\mathrm{T}}$ were the average of the $\mathrm{np}$ and $\mathrm{pp}$ values listed in table 10 .

As in the calculation of the deuteron inelastic screening we used only the "diffractive" or energy-independent part of $\mathrm{d}^{2} \sigma / \mathrm{d} M^{2} \mathrm{~d} t$. This was obtained from Fermilab data [41] for the reactions $p+p \rightarrow p+X$ and $p+d \rightarrow d+X$. The latter data, after being corrected approximately for binding of the deuteron, provided a very convenient average of the $n p$ and $p p$ inclusive production cross sections. The results of the two experiments are reasonably consistent and were combined to obtain an overall fit. The data are well represented by the form

$$
\frac{\mathrm{d}^{2} \sigma}{\mathrm{d} M^{2} \mathrm{~d} t}=A\left(M^{2}\right) \mathrm{e}^{B\left(M^{2}\right) t},
$$

where $A$ and $B$ are not functions of energy. A reasonable fit to the data is given by 


$$
\begin{aligned}
& A\left(M^{2}\right)=26.470\left(M^{2}-1.17\right)-35.969\left(M^{2}-1.17\right)^{2}+18.470\left(M^{2}-1.17\right)^{3} \\
& -4.143\left(M^{2}-1.17\right)^{4}+0.341\left(M^{2}-1.17\right)^{5} \text { for } 1.17<M^{2}<5 \mathrm{GeV}^{2} \\
& =4.4 / M^{2} \text { for } M^{2}>5 \mathrm{GeV}^{2}
\end{aligned}
$$

with $A$ in $\mathrm{mb} /(\mathrm{GeV})^{4}$, and

$$
\begin{aligned}
& B\left(M^{2}\right)=20.72-4.368\left(M^{2}-1.17\right) \\
& +0.318\left(M^{2}-1.17\right)^{2} \text { for } 1.17<M^{2}<6 \mathrm{GeV}^{2} \\
& =6.6 \mathrm{GeV}^{-2} \text { for } M^{2}>6 \mathrm{GeV}^{2} .
\end{aligned}
$$

Values of $\mathrm{d}^{2} \sigma(t=0) / \mathrm{d} M^{2} \mathrm{~d} t=A\left(M^{2}\right)$ from eq. (36) were used in evaluating $\Delta_{\text {inel }}$ for both deuterons and heavy nuclei. The uncertainty in the normalization of the experimental data leads to an overall uncertainty $\sim 20 \%$ in $A\left(M^{2}\right)$. The triple integral involved in evaluating eq. (33) was done numerically to an accuracy $\approx 1 \%$. The values we obtain for $\Delta_{\text {inel }}\left(P_{\text {lab }}\right)$ are typically within $10 \%$ of those given by Karmanov and Kondratyuk [43] for $P_{\text {lab }}>80 \mathrm{GeV} / c$, but are significantly smaller than theirs at lower momenta. Theirs were calculated before the Fermilab inclusive production cross sections were available. Their choice of $A\left(M^{2}\right)$ is not given in their article, so a direct comparison of our calculation with theirs is not possible.

Cross sections calculated with and without the inelastic screening corrections are plotted along with the experimental results in figs. 13a and 13b. The nuclear radii were chosen to give good agreement between theory and experiment below $10 \mathrm{GeV} / c$. The curves with the inelastic screening generally agree very well with the data over the entire momentum range $4<P_{\text {lab }}<300 \mathrm{GeV} / c$. If the inelastic screening is not included, there is a serious disagreement at high momenta. The agreement when inelastic screening is included is especially impressive when one recalls that the theoretical curves were made with only one fitted parameter, the nuclear half-density radius $R$. The slight discrepancy in the case of copper could be removed by a one standard deviation shift of the $5.7 \mathrm{GeV} / c$ point. The overall agreement would be improved somewhat if the inelastic screening corrections were increased $10-15 \%$. This is well within the $\pm 40 \%$ uncertainty we estimate for the calculation.

In table 11 we show the sensitivity of the calculated cross sections to changes in the input parameters. The variations of $\pm 1 \mathrm{mb}$ in $\sigma_{\mathrm{T}}(\mathrm{NN}), \pm 0.1$ in $\bar{\alpha}$, and \pm 1 in $\bar{\beta}$ can be considered as generous estimates of the typical uncertainty in these quantities. Table 11 also shows the inelastic screening correction at $300 \mathrm{GeV} / c$ for comparison.

In table 12 we compare our values for $R$ with previous determinations of nuclear radii. In this comparison we have to keep in mind an important caveat. We did not include in our calculations the effect of correlations within the nucleus. The magnitude and even the sign of these are poorly known. This could lead to an (energy independent) error $\sim 1 \%$ in the calculated total cross sections [5a] and a corresponding 
Table 11

Sensitivity of the calculated cross sections to changes in input parameters; the inelastic screening corrections at $300 \mathrm{GeV} / c$ (as a percentage of the total cross section) are shown for comparison

Nucleus $\frac{\Delta \sigma_{\text {inel }}}{\sigma} \quad \Delta \mathrm{R}= \pm 0.1 \mathrm{fm} \quad \Delta \sigma_{\mathrm{NN}}= \pm 1 \mathrm{mb} \quad \Delta \bar{\alpha}= \pm 0.1 \quad \Delta \bar{\beta}= \pm 1 \mathrm{Gev}^{2}$

$\begin{array}{lrrrrr}\text { Be } & 5.1 \% & \pm 0.8 \% & \pm 2.0 \% & \mp 0.2 \% & \pm 0.3 \% \\ \mathrm{C} & 5.8 \% & 1.0 \% & 1.8 \% & 0.2 \% & 0.3 \% \\ \mathrm{Al} & 6.4 \% & 1.5 \% & 1.5 \% & 0.4 \% & 0.4 \% \\ \mathrm{Cu} & 6.6 \% & 1.8 \% & 1.2 \% & 0.3 \% & 0.4 \% \\ \mathrm{~Pb} & 5.3 \% & 1.7 \% & 0.8 \% & 0.3 \% & 0.3 \%\end{array}$

\section{Table 12}

Our values for the half-density radii $R$ compared to results of previous measurements of nuclear radii; those of Alvensleben et al. are "strong interaction radii"; the r.m.s. radii are given to facilitate comparison

\begin{tabular}{|c|c|c|c|c|c|c|c|c|c|}
\hline \multirow[b]{2}{*}{ Nucleus } & \multicolumn{3}{|c|}{ Alvensleben et al (a) } & \multicolumn{3}{|c|}{ This Exp't } & \multicolumn{3}{|c|}{ Electromagnetic ${ }^{(b)}$} \\
\hline & $\mathrm{R}$ & $\mathbf{s}$ & $\mid\left(r^{2}\right\rangle^{\frac{1}{2}}$ & $\mathbf{R}$ & $\mathbf{s}$ & $\left\langle\mathrm{r}^{2}\right\rangle^{\frac{1}{2}}$ & $\mathbf{R}$ & 8 & $\left\langle r^{2}\right\rangle^{\frac{1}{2}}$ \\
\hline $\mathrm{Be}^{9}$ & $\begin{array}{r}2.35 \\
\pm .25\end{array}$ & $2.4^{\star}$ & $\begin{array}{l}2.72 \\
\pm .13\end{array}$ & $\begin{array}{l}2.10 \\
\pm .25\end{array}$ & $2.3^{*}$ & $\begin{array}{r}2.53 \\
\pm .13\end{array}$ & $\begin{array}{r}1.80 \\
\pm .08\end{array}$ & $\begin{array}{l}2.0 \\
\pm .15\end{array}$ & 2.19 \\
\hline$c^{12}$ & $\begin{array}{l}2.50 \\
\pm .23\end{array}$ & $2.4^{\star}$ & $\begin{array}{l}2.80 \\
\pm .12\end{array}$ & $\begin{array}{l}2.13 \\
\pm .19\end{array}$ & $2.3^{*}$ & $\begin{array}{r}2.55 \\
\pm .10\end{array}$ & $\begin{array}{r}2.30 \\
\pm .09\end{array}$ & $\begin{array}{r}1.85 \\
\pm .15\end{array}$ & 2.37 \\
\hline $\mathrm{Al}^{27}$ & $\begin{array}{r}3.37 \\
\pm .16\end{array}$ & $2.4^{\star}$ & $\begin{array}{l}3.30 \\
\pm .10\end{array}$ & $\begin{array}{r}3.11 \\
+.14\end{array}$ & $2.3^{\star}$ & $\begin{array}{l}3.10 \\
\pm .09\end{array}$ & $\begin{array}{r}3.07 \\
\pm .09\end{array}$ & $\begin{array}{l}2.28 \\
\pm .11\end{array}$ & 3.06 \\
\hline $\mathrm{Cu}^{54}$ & $\begin{array}{l}4.55 \\
\pm .11\end{array}$ & $2.4^{*}$ & $\begin{array}{l}4.07 \\
\pm .07\end{array}$ & $\begin{array}{l}4.49 \\
\pm .11\end{array}$ & $2.3^{\star}$ & $\begin{array}{r}3.99 \\
\pm .07\end{array}$ & $\begin{array}{l}4.15 \\
\pm .10\end{array}$ & $2.5^{*}$ & $\begin{array}{l}3.85 \\
\pm .05\end{array}$ \\
\hline $\mathrm{Pb}^{208}$ & $\begin{array}{l}5.82 \\
\pm .20\end{array}$ & $2.4^{*}$ & $\begin{array}{l}5.55 \\
\pm .15\end{array}$ & $\begin{array}{r}5.80 \\
\pm .12\end{array}$ & $2.3^{*}$ & $\begin{array}{l}5.62 \\
\pm .09\end{array}$ & $\begin{array}{l}6.50 \\
\pm .10\end{array}$ & $\begin{array}{r}2.30 \\
\pm .15\end{array}$ & 5.40 \\
\hline
\end{tabular}

${ }^{\star}$ Not a free parameter.

(a) H. Alvensleben et al., Phys. Rev. Lett. 24, 792 (1970).

(b) L. Elton, Nuclear Sizes, Oxford Univ, Press, London, 1961.

H. Thiessen et al., Z. Physik, 231, 475 (1970).

R. Lombard and G. Bishop, Nucl, Phys. Al01, 60i (1967). 
error $\sim 0.1 \mathrm{fm}$ in $R$. In addition, we have kept the skin thickness of all nuclei fixed at $2.3 \mathrm{fm}$.

We have assigned a nominal uncertainty to our values of $R$ by assuming an overall uncertainty of $\pm 2 \%$ in the total cross-section data and in the calculated cross sections. Our values for $\left\langle r^{2}\right\rangle^{\frac{1}{2}}$ lie closer to the electromagnetic radii than those of Alvensleben et al. The latter are strong interaction radii determined from data on the photoproduction of $\rho$ mesons on nuclei.

\section{Conclusions}

We have measured total cross sections for neutrons on protons, deuterium, beryllium, carbon, aluminum, iron, copper, cadmium, tungsten, lead and uranium at 7 momenta between 30 and $300 \mathrm{GeV} / c$. Typical accuracy of the data is 0.5 to $1 \%$.

On the basis of our results and those of previous experiments the cross sections are consistent with an $A^{0.77 \pm 0.01}$ dependence for momenta above $5 \mathrm{GeV} / c$.

The cross sections for deuterium and heavy nuclei are consistent with theoretical predictions only if inelastic screening is included. Expressions given by Gribov [32] for deuterium and by Karmanov and Kondratyuk [43] for heavy nuclei seem to give reasonably accurate estimates for the inelastic screening effect.

The overall agreement between the more recent neutron-nucleus total cross section measurements [4-7] is very good. The agreement with theory is also impressive.

As a byproduct of our analysis, we obtain nuclear radii in good agreement with previous determinations by other techniques.

It is a pleasure to take this opportunity to thank the many people who made numerous vital contributions to the experiment. The experiment would not have been possible without the dedicated efforts of the Fermilab staff. James Stone, John Chanowski, Orman Haas, James Pluta, David Burke, and Donald Koch assisted in the assembly and testing of the equipment. Frederick Ringia, Clark deHaven, and Richard Schultz made major contributions to the programming and data analysis.

Bruce Cork and Oliver Overseth participated in many phases of the experiment. We are especially grateful to Thomas Mc Corriston for providing computer programs and advice in calculating theoretical cross sections. Helpful conversations with Gordon Kane are gratefully acknowledged.

\section{References}

[1] M.J. Longo et al., Phys. Rev. Letters 33 (1974) 725.

[2] L.W. Jones et al., Phys. Rev. Letters 33 (1974) 1440.

[3] M.N. Kreisler et al., Phys. Rev. Letters 20 (1968) 468.

[4] E.F. Parker et al., Phys. Letters 31B (1970) 246. 
[5] (a) T.P. McCorriston, Jr., UM HE 72-11, Ph. D. thesis, University of Michigan, unpublished;

(b) L.W. Jones et al., Phys. Letters 36B (1971) 509.

[6] J. Engler et al., Phys. Letters 31 B (1970) 669;

J. Engler et al., Phys. Letters 32B (1970) 716.

[7] A. Babaev et al., Phys. Letters $51 B$ (1974) 501;

A. Babaev et al., Inst. for Theoretical and Exp. Physics report, ITEP-10, 1974, unpublished.

[8] A. Carroll et al., Phys. Rev. Letters 33 (1974) 932.

[9] S.P. Denisov et al., Nucl. Phys. B65 (1973) 1.

[10] M. Longo and O. Haas, Rev. Sci. Inst. 45 (1974) 456.

[11] L.W. Jones et al., Nucl. Instr. 118 (1974) 431.

[12] H.M. Roder et al., Survey of the properties of hydrogen isotopes below their critical temperatures, U.S. Nat'l Bureau of Standards Tech. Note No. 641 (U.S. GPO, Washington, D.C. 1973).

[13] M.J. Longo et al., Characteristics of the M-3 neutral beam at NAL, Univ. of Michigan high-energy group report UM HE 74-18, unpublished.

[14] W. Lakin et al., Phys. Letters 31B (1970) 677.

[15] B. Rossi, High-energy particles (Prentice-Hall, N.J., 1952).

[16] W. Baker et al., Phys. Letters 51B (1970) 677.

[17] Pisa-Stony Brook (ISR):

S.R. Amendolia et al., Phys. Letters 44B (1973) 119;

G. Bellettini, High-energy collisions - 1973, AIP Conf. Proc. No. 15 (AIP, New York, 1974).

[18] CERN-Rome (ISR):

U. Amaldi et al., Phys. Letters 44B (1973) 112.

[19] FNAL bubble chamber:

G. Charlton et al., Phys. Rev. Letters 29 (1972) 515;

C. Bromberg et al., Phys. Rev. Letters 31 (1973) 1563;

A. Firestone et al., Phys. Rev. D10 (1974) 2080.

[20] A. Carroll et al., Phys. Rev. Letters 33 (1974) 928.

[21] S.P. Denisov et al., Phys. Letters 36B (1971) 415.

[22] W. Galbraith et al., Phys. Rev. 138B (1965) 913.

[23] D.V. Bugg et al., Phys. Rev. 146 (1966) 980.

[24] K.J. Foley et al., Phyṣ. Rev. Letters 19 (1967) 857.

[25] H.R. Gustafson et al., Phys. Rev. Letters 32 (1974) 441.

[26] K.F. Riley, Phys. Rev. D1 (1970) 2481.

[27] J. Pumplin and M. Ross, Phys. Rev. Letters 21 (1968) 1778.

[28] B. Udgaonkar and M. Gell-Mann, Phys. Rev. Letters 8 (1962) 346.

[29] G. Kane and A. Seidl, private communication.

[30] A. Bouquet et al., A possible incomptability between the $N N$ and $\bar{N} N$ total cross sections and the Regge-pole model, Univ. of Paris report PAR-LPTHE 74-11 (Dec. 1974), unpublished.

[31] R.J. Glauber, Phys. Rev. 100 (1955) 242;

V. Franco and R.J. Glauber, Phys. Rev. 142 (1966) 1195.

[32] V.N. Gribov, ZhETF (USSR) 56 (1969) 892; JETP (Sov. Phys.) 29 (1969) 483.

[33] C. Quigg and L. Wang, Phys. Letters 43B (1973) 314.

[34] V. Anisovich et al., Phys. Letters 42B (1972) 224.

[35] A. Kaidalov and L. Kondratyuk, Nucl. Phys. B56 (1973) 90.

[36] (a) V. Bartenev et al., Phys. Rev. Letters 31 (1973) 1367;

(b) G. Beznogikh et al., Phys. Letters 39B (1972) 411;

(c) L. Kirillova et al., ZhETF (USSR) 50 (1966) 76; JETP (Sov. Phys.) 23 (1966) 52. 
[37] (a) G. Beznogikh et al., Yad. Fiz. 18 (1973) 348; Sov. J. Nucl. Phys. 18 (1974) 179.

(b) V. Franco and G. Varma, Phys. Rev. Letters 33 (1974) 44.

[38] A. Lengyel and V. Lengyel, Some consequences on the equality of pp and np scattering total cross sections, Inst. for Theoret. Physics, Kiev report ITP-74-46E, unpublished.

[39] (a) V. Bartenev et al., Phys. Rev. Letters 31 (1973) 1088;

(b) M. Perl et al., Phys. Rev. D1 (1970) 1857.

[40] G.V. Bochman et al., Phys. Letters 33B (1970) 222.

[41] (a) V. Bartenev et al., Phys. Letters 51B (1974) 299;

(b) Y. Akimov et al., Fermilab report NAL-Conf 74/56 EXP, unpublished;

(c) Y. Akimov et al., Fermilab report NAL-Conf 74/66 EXP, unpublished.

[42] V. Franco, Phys. Rev. C6 (1972) 748.

[43] V. Karmanov and L. Kondratyuk, ZhETF Pisma 18 (1973) 451; JETP Letters (Sov. Phys.) 18 (1973) 266. 